\title{
SEIFERT MATRICES AND BOUNDARY LINK COBORDISMS
}

\author{
KI HYOUNG KO
}

\begin{abstract}
To an $m$-component boundary link of odd dimension, a matrix is associated by taking the Seifert pairing on a Seifert surface of the link. An algebraic description of the set of boundary link cobordism classes of boundary links is obtained by using this matrix invariant.
\end{abstract}

1. Introduction. An $m$-link of dimension $n$ is a smooth oriented submanifold $L$ in $S^{n+2}$ where $L=L_{1} \cup \cdots \cup L_{m}$ is the ordered disjoint union of $m$ manifolds which are each homeomorphic to $S^{n} . S^{n+2}$ in the definition is assumed to be given a fixed orientation and called the ambient sphere. Two $m$-links $L_{0}, L_{1}$ are said to be cobordant if there is a smooth oriented submanifold $I L$ in $S^{n+2} \times I$ such that

(i) $I L$ intersects $S^{n+2} \times i$ transversely at $L_{i}$, for $i=0,1$;

(ii) $I L$ is homeomorphic to $L_{0} \times I$.

$I L$ is called a link cobordism of $L_{0}$ to $L_{1}$. Let $C(n, m)$ denote the set of cobordism classes of $m$-links of dimension $n$. Then $C(n, 1)$ is the usual knot cobordism group.

There have been at least three effective ways to study knot cobordisms. The first approach is to use the Seifert pairing which is defined on the middle dimensional homology of a manifold bounded by an odd dimensional knot [L3, L4]. Another approach which is closely related to the first, is to use the Blanchfield pairing which is defined on the middle dimensional knot module $[\mathbf{K 1} \mathbf{1}$. These two approaches are intuitive and have the advantage that they classify odd dimensional simple knots up to ambient isotopy as well [L5, K2]. The third approach is to construct and classify the complements of knot cobordisms using homology surgery [CS1]. Since this approach relies on a general surgery machine, it can be applied to more general situations [CS1, CS and De]. All these three approaches completely solve the higher dimensional knot cobordism problem. An equivalence between the first two pairings has been given explicitly in [L6] and, between the last two approaches, abstractly in [Ra].

One might start to study the link cobordism problem by asking whether the problem can be reduced to the knot cobordism problem, that is, whether $C(n, m)$ is isomorphic to $m C(n, 1)$, the direct sum of $m$ copies of $C(n, 1)$. The answer is, unfortunately, no [CS]. The direct attack on $C(n, m)$ using Seifert pairings faces an immediate difficulty that one cannot find a "nice" manifold bounded by a link which has enough information to work with. So we will consider, rather, the following kind of $m$-links. An $m$-link $L$ in $S^{n+2}$ is called a boundary link if it bounds $m$ disjoint orientable submanifolds in $S^{n+2}$. We define a natural relation called boundary link cobordism between boundary links. Two boundary $m$-links $L_{0}$,

Received by the editors June 7, 1985 and, in revised form, February 20, 1986.

1980 Mathematics Subject Classification (1985 Revision). Primary 57C45; Secondary $57 \mathrm{M} 25,57 \mathrm{Q} 60,57 \mathrm{R} 65$.

Key words and phrases. Boundary link, boundary link cobordism, Seifert matrix. 
$L_{1}$ are boundary cobordant if there is a link cobordism $I L$ of $L_{0}$ to $L_{1}$ which bounds $m$ disjoint orientable submanifolds in $S^{n+2} \times I$. Let $B(n, m)$ denote the set of boundary cobordism classes of boundary links. It is known that $B(n, 1)=C(n, 1)$ and $B(n, m)=0$ for $n$ even $[\mathbf{K}, \mathbf{G u}]$. The difficulty above will be isolated by breaking the link cobordism problem into the following problems:

(i) Determine $B(n, m)$.

(ii) Compare boundary link cobordism with link cobordism.

(iii) Determine when a link is cobordant to a boundary link.

Problems (ii) and (iii) will not be considered in this work and one can find partial answers in $[\mathbf{C S}]$ for (ii), and in [De, Sa, Co and $\mathbf{O r}]$ for (iii).

To study $B(n, m)$ one has to deal with another difficulty which does not occur for $C(n, 1)$. The union of $m$ disjoint orientable submanifolds bounded by a boundary $m$-link $L$ is called a Seifert surface for $L$. One can choose many distinct Seifert surfaces for a given boundary link. If $m=1$ (or $m=2$, it turns out), then they are cobordant relative boundary. But this is not the case in general. Since the Seifert pairing is taken from a Seifert surface, it depends on the choice of Seifert surfaces for a given link and so we should consider a pair $(L, M)$ of a boundary link $L$ and its Seifert surface rather than a boundary link $L$ itself. A similar boundary cobordism relation on these pairs is defined and $C_{n}\left(B_{m}\right)$ denotes the set of such equivalence classes. Then the boundary connected sum gives an abelian group structure on $C_{n}\left(B_{m}\right)$. The change of choice of Seifert surfaces for a given boundary $m$-link can be described as an action by the group $A_{m}$ of generator conjugating automorphisms of the free group with $m$ generators. S. Cappell and J. Shaneson $[\mathbf{C S}]$ have alternatively considered pairs $(L, \theta)$ called $F_{m}$-links, that is, $L$ is a boundary $m$-link and $\theta$ is a splitting map for a meridian map of the link group of $L$. A suitable cobordism relation, called $F_{m}$-cobordism, between $F_{m^{-}}$ links was defined and $C_{n}\left(F_{m}\right)$ denoted the set of $F_{m}$-cobordism classes. Then they determined $C_{n}\left(F_{m}\right)$ and $B(n, m)$ in terms of their $\Gamma$-groups $[\mathbf{C S}, \mathbf{C S 1}$ ]. In $\S 2$, we prove that there is an $1: 1$ correspondence between $C_{n}\left(B_{m}\right)$ and $C_{n}\left(F_{m}\right)$ which preserves the $A_{m}$-action.

In $\S 3$, we associate to a Seifert surface of a boundary $m$-link of odd dimension a matrix which represents the Seifert pairing on the Seifert surface. Such a matrix which has $m^{2}$ integral matrices as entries is called a Seifert matrix. Let $G(m, \varepsilon)$ be the abelian group of cobordism classes of Seifert matrices of type $(m, \varepsilon), \varepsilon= \pm 1$. An action of $A_{m}$ on $G(m, \varepsilon)$ is also defined. Then we have the following algebraic formulations of $B(n, m)$. For $n=2$ or $n \geq 4, B(n, m)$ is isomorphic to

$$
\begin{cases}0 & \text { if } n \equiv 0(\bmod 2), \\ G(m,+) / A_{m} & \text { if } n \equiv 1(\bmod 4) \\ G(m,-) / A_{m} & \text { if } n \equiv 3(\bmod 4)\end{cases}
$$

and $B(3, m)$ is isomorphic to $G_{0}(m,+) / A_{m}$ where $G_{0}(m,+)$ is a subgroup of index $2^{m}$ in $G(m,+)$.

In $\S 4$, we give an example of a boundary link bounding two Seifert surfaces that are not boundary cobordant.

This work is based on a part of the author's Ph.D. thesis, written at Brandeis University. I am grateful for the guidance of Professor J. Levine. 
2. Boundary links and boundary link cobordisms. An $m$-link $L=L_{1} \cup$ $\cdots \cup L_{m}$ in $S^{n+2}$ is called a boundary link if $L_{i}$ is the oriented boundary of a smooth, oriented submanifold $M_{i}$ in $S^{n+2}$ for each $i=1, \ldots, m$ and $M_{1}, \ldots, M_{m}$ are disjoint. The ordered union $M=M_{1} \cup \cdots \cup M_{m}$ is called a Seifert surface ${ }^{1}$ of $L$ if each $M_{i}$ is connected. One can choose infinitely many topologically distinct Seifert surfaces for a given link.

By using the trivial normal bundle of $L$ in $S^{n+2}$, we can find a tubular neighborhood $T$ of $L$ which is diffeomorphic to $L \times D^{2}$ and unique up to ambient isotopy relative $L$ for $n \geq 2$. Let $X$ be the closure of $S^{n+2}-T$, which will be called an exterior of $L$. When we identify $\partial X$ with $L \times S^{1}$, the orientation of $L$ and the fixed orientation of the ambient sphere determine the oriented meridian fiber on the $i$ th component of $\partial X$, i.e., $p \times S^{1}$ for $p \in L_{i}$. If we connect it to the base point in $X$ by a path, we have an element in $\Pi_{1} X$ which is called an $i$ th meridian in $\Pi_{1} X$ and is well defined up to conjugation.

$F_{m}$ denotes the free group generated by $m$ letters $x_{1}, \ldots, x_{m}$. The following proposition is due to Smythe $[\mathbf{S m}]$ and Gutierrez $[\mathbf{G u}]$. We will give a proof which will be needed for Lemma 2.2 .

Proposition 2.1. L is a boundary m-link in $S^{n+2}$ if and only if there is an epimorphism $\theta: \Pi_{1} X \rightarrow F_{m}$ which sends an ith meridian to $x_{i}$ for $i=1, \ldots, m$.

Proof. Let $M$ be a Seifert surface of $L$ and $\bar{M}=M \cap X$. The ThomPontryagin construction of submanifold pair in $(\bar{M}, \partial \bar{M})$ in $(X, \partial X)$ gives a map $f: X \rightarrow \bigvee S^{1}$ where $\bigvee S^{1}$ is the $m$-fold wedge product of $S^{1}$.

By specifying base points of each copy of $m\left(S^{n} \times S^{1}\right)$, we have a map from $m$ points to $m\left(S^{n} \times S^{1}\right)$. Let $A$ be the mapping cone of this map. Since $\bar{M}$ is a disjoint union of connected bounded submanifolds, $X-\bar{M}$ is (pathwise) connected. So we can find $m$ paths from the base point (not in $\bar{M}$ ) of $X$ to each component of $\partial X$. This defines an embedding

$$
i:\left(A, m\left(S^{n} \times S^{1}\right), \text { the cone point }\right) \rightarrow(X, \partial X, \text { the base point }) .
$$

We identify $\Pi_{1} A$ and $\Pi_{1} \bigvee S^{1}$ with $F_{m}$ in the obvious way. Then the composite $f_{*} i_{*}$ of the induced maps on the fundamental group is the identity map of $F_{m}$.

Conversely, let $\mu: F_{m} \rightarrow \Pi_{1} X$ be a homomorphism sending $x_{i}$ to an $i$ th meridian whose image is $x_{i}$ under $\theta$ for each $i$. We realize $\mu$ by an embedding $i: A \rightarrow X$. The projections on each copy $S^{n} \times S^{1}$ of $A$ define a map $e: A \rightarrow \bigvee S^{1}$ by sending all arcs of $A$ to the base point of $\bigvee S^{1}$. Since we have on fundamental groups that $e_{*}=$ id, $i_{*}=\mu$ and $\theta \mu=\mathrm{id}$, there is an extension $f: X \rightarrow \bigvee S^{1}$ of $e$ such that $f_{i}=e$ and $f_{*}=\theta$. The transversality of $f$ to $m$ disjoint points, one from each circle of $\bigvee S^{1}$, gives $m$ disjoint submanifolds $M_{1}, \ldots, M_{m}$. We note that since the restriction $f \mid \partial X$ behaves like the map projecting the $i$ th copy $S^{n} \times S^{1}$ of $m\left(S^{n} \times S^{1}\right)$ to the $i$ th $S^{1}$ of $\bigvee S^{1}$, the $i$ th copy of $\partial X$ contains only $\partial M_{i}$. Each $M_{i}$ is not necessarily connected. We may throw away closed components from $M=M_{1} \cup \cdots \cup M_{m}$ so that we have a Seifert surface for $L$ by adding collars to $M$.

Such an epimorphism $\theta$ will be called a splitting map for $L$ because it makes a meridian map $\mu: F_{m} \rightarrow \Pi_{1} X$ sending $x_{i}$ to an $i$ th meridian for each $i$ split, i.e., $\theta \mu=$ id.

\footnotetext{
${ }^{1}$ Although $n \geq 2$, it is a tradition to use this terminology of the classical dimensional analogue after $[$ Se].
} 
In the last argument of the proof of 2.1 , the arbitrary removal of closed components costs us that the resulting Seifert surface may not induce the given splitting map $\theta$. For a later purpose, we need

LEMMA 2.2. For a given splitting map $\theta$ of a boundary link $L$, there is a Seifert surface of $L$ which induces $\theta$ by the Thom-Pontryagin construction.

PROOF. In the proof of 2.1 above we had submanifolds $M_{1}, \ldots, M_{m}$. The $i$ th arc of $i(A)$ in $X$ may intersect $M_{1}, \ldots, M_{m}$. We write down the intersection as a word $w_{i}$ on letters $x_{1}, \ldots, x_{m}$ in the obvious way. Then the $i$ th generator of $\Pi_{1} A$ goes to $w_{i} x_{i} w_{i}^{-1}$ under the map $e_{*}: \Pi_{1} A \rightarrow F_{m}$. Since $e_{*}=\theta \mu=$ id when we identify $\Pi_{1} A$ with $F_{m}$, the word $w_{i} x_{i} w_{i}^{-1}$ should be reducible to $x_{i}$. Thus $w_{i}$ must be reduced to a product of $x_{i}$ (or $x_{i}^{-1}$ ) by cancelations. We realize these cancelations by connected sum, that is, if $w_{i}$ contains a subword of the form $x_{k} x_{k}^{-1}$ (or $x_{k}^{-1} x_{k}$ ) for some $k=1, \ldots, m$ then $M_{k}$ meets the $i$ th arc of $i(A)$ twice successively with different signs. We do an index 1 ambient surgery using the tube, homeomorphic to $D^{1} \times S^{n}$ around the $i$ th arc. The orientation extends to the tube. We continue this process for all cancelations in $w_{i}$. One has to use a thinner tube to realize another cancelation through the cancelation which has been already realized, e.g., $w_{i}=x_{j}^{-1} x_{k} x_{k}^{-1} x_{j}$. Now the $i$ th arc intersects only $M_{i}$ a number of times. We do this for all $w_{i}, i=1, \ldots, m$, to get $m$ new submanifolds $M_{1}^{\prime}, \ldots, M_{m}^{\prime}$ such that $M_{i}^{\prime}$ intersects only the $i$ th arc for each $i$. The Thom-Pontryagin construction of $M_{1}^{\prime} \cup \cdots \cup M_{m}^{\prime}$ gives a map $f^{\prime}: X \rightarrow \bigvee S^{1}$ which is homotopic to $f$. We now get rid of closed components from $M_{1}^{\prime}, \ldots, M_{m}^{\prime}$, if there are any. So we have connected submanifolds $M_{1}^{\prime \prime} \cup \cdots \cup M_{m}^{\prime \prime}$ in $X$. Let $f^{\prime \prime}: X \rightarrow \bigvee S^{1}$ be the map obtained from $M_{1}^{\prime \prime} \cup \cdots \cup M_{m}^{\prime \prime}$. Then $f_{*}^{\prime} i_{*}=f_{*}^{\prime \prime} i_{*}$ on fundamental groups. We appeal to a Stalling's theorem $[\mathbf{S t}]$ to show $f_{*}^{\prime \prime}=f_{*}^{\prime}$ and so $f_{*}^{\prime \prime}=\theta$. Since $H_{1}\left(\Pi_{1} X\right)=H_{1}\left(F_{m}\right)=\mathbf{Z}^{m}$ and $H_{2}\left(F_{m}\right)=0, f_{*}^{\prime}, f_{*}^{\prime \prime}$ and $i_{*}$ induce isomorphisms and the commutative diagram:

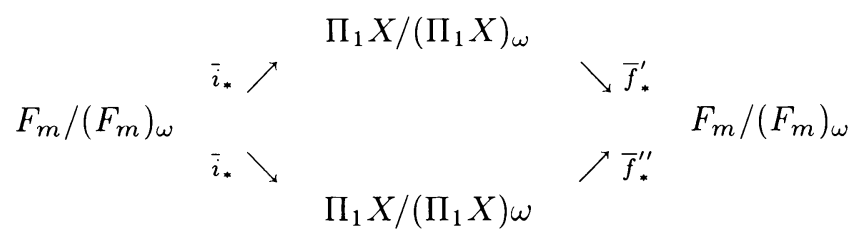

where $G_{\omega}$ denotes the intersection of terms in the lower central series of $G$. Then $\bar{f}_{*}^{\prime}=\bar{f}_{*}^{\prime \prime}$. Since $\left(F_{m}\right)_{\omega}$ is the trivial group, we have ker $f_{*}^{\prime}=\left(\Pi_{1} X\right)_{\omega}=\operatorname{ker} f_{*}^{\prime \prime}$. Thus $f_{*}^{\prime \prime}=f_{*}^{\prime}$. This shows that $M_{1}^{\prime \prime} \cup \cdots \cup M_{m}^{\prime \prime}$ is the desired Seifert surface of $\theta$.

Let $C A_{m}$ be the group of automorphisms of $F_{m}$ which send $x_{i}$ to a conjugate of $x_{i}$ for each $i=1, \ldots, m$. The following lemma has been observed in $[\mathbf{C S}]$.

LEMMA 2.3. Let $L$ be a boundary $m$-link in $S^{n+2}$.

(1) If $\theta$ is a splitting map for $L$, then so is $\alpha \cdot \theta$ for all $\alpha \in C A_{m}$.

(2) If $\theta_{1}$ and $\theta_{2}$ are splitting maps then $\theta_{2}=\alpha \cdot \theta_{1}$ for some $\alpha \in C A_{m}$.

The above lemma shows that all splitting maps of a given boundary link can be found by compositions by elements of $C A_{m}$ with a given splitting map. In order to describe the corresponding geometric changes of a given Seifert surface, we will use the following lemma. 
Let $\alpha \in C A_{m}$, then for each $i=1, \ldots, m, \alpha\left(x_{i}\right)=w_{i} x_{i} w_{i}^{-1}$ for some $w_{i} \in F_{m}$. We write simply $\alpha=\left(w_{1}, \ldots, w_{m}\right)$. This expression is unique if each $w_{i} x_{i} w_{i}^{-1}$ is (freely) reduced, i.e., $w_{i}$ is (freely) reduced and does not end with the letter $x_{i}$ or $x_{i}^{-1}$.

We define elements $\alpha_{i j}=\left(e, \ldots, e, x_{j}, e, \ldots, e\right)$ where $x_{j}$ is in the $i$ th spot and $e$ denotes the empty word. Note that $\alpha_{i j}^{-1}=\left(e, \ldots, e, x_{j}^{-1}, e, \ldots, e\right)$ where $x_{j}^{-1}$ is in the $i$ th spot.

LEMMA 2.4. $C A_{m}$ is generated by $\alpha_{i j}$ for $i, j=1, \ldots, m$ and $i \neq j$.

PROOF. It is easy to see that $C A_{m}$ is a group. In fact, if $\alpha=\left(w_{1}, \ldots, w_{m}\right)$ then $\alpha^{-1}=\left(\alpha^{-1}\left(w_{1}^{-1}\right), \ldots, \alpha^{-1}\left(w_{m}^{-1}\right)\right)$. For $\alpha \in C A_{m}$, we define the length $l(\alpha)$ of $\alpha$ by $l(\alpha)=l\left(w_{1}\right)+\cdots+l\left(w_{m}\right)$ after we write $\alpha=\left(w_{1}, \ldots, w_{m}\right)$ uniquely where $l(w)$ is the length of a reduced word $w$. Then one can easily see that $l(\alpha)=0$ if and only if $\alpha=$ id. Let $\alpha$ be an element in $C A_{m}$ which is not the identity. We write $\alpha=\left(w_{1}, \ldots, w_{m}\right)$ in the unique way. Then we claim that there is at least one pair $(j, k)$ for some $j, k=1, \ldots, m$ such that $w_{k}=w_{j} x_{j}^{\varepsilon} \bar{w}_{k}$ where $\bar{w}_{k}$ is a reduced word which does not end with $x_{k}$ or $x_{k}^{-1}$ and $\varepsilon= \pm 1$. Since $w_{k}$ was reduced, $\bar{w}_{k}$ can not start with $x_{j}^{-\varepsilon}$. Then $\alpha \alpha_{k j}^{-\varepsilon}=\left(w_{1}^{\prime}, \ldots, w_{m}^{\prime}\right)$ where $w_{i}^{\prime}=w_{i}$ for $i \neq k$ and $w_{k}^{\prime}=w_{j} \bar{w}_{k}$. So $l\left(\alpha \alpha_{k j}^{-\varepsilon}\right)$ is strictly less than $l(\alpha)$. By induction on the length of automorphism, we can make $\alpha$ into the identity by composing $\alpha_{i j}^{\varepsilon}$ 's on the right hand side. To show our claim, we write $\alpha^{-1}=\left(u_{1}, \ldots, u_{m}\right)$ uniquely. Since $\alpha \neq$ id, there is a $k=1, \ldots, m$ such that $w_{k}$ is not the empty word and neither is $u_{k}$. If $u_{k}$ is given by the word $x_{k_{1}}^{\varepsilon_{1}} \cdots x_{k_{r}}^{\varepsilon_{r}}$, then

$$
x_{k}=\alpha \alpha^{-1}\left(x_{k}\right)=\left(\prod_{s=1}^{r}\left(w_{k_{s}} x_{k_{s}}^{\varepsilon_{s}} w_{k_{s}}^{-1}\right)\right) w_{k} x_{k} w_{k}^{-1}\left(\prod_{s=1}^{r}\left(w_{k_{s}} x_{k_{s}}^{\varepsilon_{s}} w_{k_{s}}^{-1}\right)\right)^{-1} .
$$

Since $w_{i} x_{i} w_{i}^{-1}$ was a reduced word for each $i=1, \ldots, m$, so is $w_{k_{s}} x_{k_{s}}^{\varepsilon_{s}} w_{k_{s}}^{-1}$ for each $s=1, \ldots, r$. Thus in the above product any cancelation must begin between one of pairs $\left(w_{k_{r}}^{-1}, w_{k}\right)$ or $\left(w_{k_{s}}^{-1}, w_{k_{s+1}}\right)$ for $s=1, \ldots, r-1$. We now assume that our claim is false. Then there is no way for any of the middle letters in the block, i.e., $x_{k}$ and $x_{k_{1}}^{\varepsilon_{1}}, \ldots, x_{k_{r}}^{\varepsilon_{r}}$ in the above product, to be canceled out. Since there is at least one letter among these middle letters which is different from $x_{k}$ or $x_{k}^{-1}$ (for example $\left.x_{k_{r}}^{\varepsilon_{r}}\right)$, this is a contradiction.

We are going to find invariants ${ }^{2}$ extracted from Seifert surfaces rather than boundary links themselves. Seifert surfaces of a given knot are all cobordant relative their boundaries. So one can analyze them by using the Morse theory [L5]. But this is not the case for boundary links due to the fact that splitting maps of a given link may differ by more than inner automorphisms, i.e., elements in $C A_{m}$. One can easily see that if $m \geq 3, C A_{m}$ contains elements which are not inner. Thus it is natural to consider the following set.

\footnotetext{
${ }^{2}$ Cappell and Shaneson have used the terminology "local" for these invariants, comparing with their approaches analyzing complements of links [CS].
} 
Let $S(n, m)$ denote the set of all Seifert surfaces with $m$ components in $S^{n+2}$, that is, $M \in S(n, m)$ if and only if

(i) $\partial M$ is homeomorphic to $m$ copies of $S^{n}$;

(ii) $M$ is a disjoint ordered union of oriented connected submanifolds $M_{1}, \ldots, M_{m}$ in $S^{n+2}$.

The orientation of $\partial M$ or $M$ determines a normal vector field of $M$ in $S^{n+2}$, called the positive normal direction of $M$, of the trivial normal bundle of $M$ in $S^{n+2}$, and vice versa.

We define the following relation on the set $S(n, m)$. Let $M, N$ be in $S(n, m) . M$ and $N$ are said to be boundary cobordant if there is a submanifold $I M$ of $S^{n+2} \times I$ such that

(i) $I M \cap S^{n+2} \times 0=M$ and $I M \cap S^{n+2} \times 1=N$;

(ii) $\partial I M-\operatorname{int}(M \cup N)$ is homeomorphic to $m$ copies of $S^{n} \times I$;

(iii) $I M$ is a disjoint ordered union of oriented connected submanifolds $I M_{1}, \ldots$, $I M_{m}$ of $S^{n+2} \times I$ with the positive normal direction which restricts to the positive normal directions of $M$ and $N$ on $S^{n+2} \times 0$ and $S^{n+2} \times 1$, respectively.

Such a submanifold $I M$ will be called a Seifert surface of a boundary link cobordism, or simply a boundary cobordism. And $\partial I M-\operatorname{int}(M \cup N)$ is called a boundary link cobordism between boundary links $\partial M$ and $\partial N$.

One can check that the boundary cobordism is an equivalence relation on $S(n, m)$. We define $C_{n}\left(B_{m}\right)$ to be the set of boundary cobordism classes of Seifert surfaces in $S(n, m)$.

We define an action of $C A_{m}$ on $C_{n}\left(B_{m}\right)$ as follows: Let $\alpha_{i j}$ be one of generators of $C A_{m}$ as in Lemma 2.4. And let $M=M_{1} \cup \cdots \cup M_{m}$ be a Seifert surface in $S^{n+2}$. We place a copy $M_{i}^{+}$of $M_{i}$ parallel to $M_{i}$ in a neighborhood of $M_{i}$ on the side of the positive normal direction of $M_{i}$. Similarly we place a copy $M_{i}^{-}$ on the side of the negative normal direction. We may assume that $M_{i}^{+} \cap M$ and $M_{i}^{-} \cap M$ are empty. It is easy to see that $\partial M_{i}^{+}$and $\partial M_{i}^{-}$bounds a submanifold $V$ in $S^{n+2}-\left(M \cup M_{i}^{+} \cup M_{i}^{-}\right)$, homeomorphic to $D^{1} \times S^{n}$. Let $M_{j}^{\prime}=M_{i}^{+} \cup V \cup M_{i}^{-}$. After rounding the corners, $M_{j}^{\prime}$ is a closed submanifold in $S^{n+2}-M$. We choose an arc starting in the negative normal direction from an interior point of $M_{j}$ and ending at a point of $M_{j}^{\prime}$. Using this arc, we take an interior connected sum of $M_{j}$ and $M_{j}^{\prime}$, and denote it by $M_{j}^{\prime \prime}$. We smooth the corner of $M_{j}^{\prime \prime}$. A positive normal direction of $M_{j}^{\prime \prime}$ is induced from the one on $M_{j}$. We define an action by $\alpha_{i j}$ on $M$ by

$$
\alpha_{i j} \cdot M=M_{1} \cup \cdots \cup M_{j-1} \cup M_{j}^{\prime \prime} \cup M_{j+1} \cup \cdots \cup M_{m} .
$$

Obviously, $\alpha_{i j} \cdot M$ depends on the choice of arcs. But this action is well defined on $C_{n}\left(B_{m}\right)$. To see this, let $M, N$ be Seifert surfaces in $S(n, m)$ such that they are boundary cobordant by a boundary cobordism $I M$ in $S^{n+2} \times I$. Construct $M_{j}^{\prime}$ and $N_{j}^{\prime}$ from $M$ and $N$ as above. Similarly, construct a submanifold $I M_{j}^{\prime}$ in $\left(S^{n+2} \times I\right)-I M$ surrounding $I M_{i}$ so that $\partial I M_{j}^{\prime}=M_{j}^{\prime} \cup N_{j}^{\prime}$. We take a boundary connected sum of $I M_{j}^{\prime}$ and $I M_{j}$ in $S^{n+2} \times 0$ using the arc which has been used for the interior connected sum of $M_{j}^{\prime}$ and $M_{j}$. And we simultaneously take another boundary connected sum of $I M_{j}^{\prime}$ and $I M_{j}$ in $S^{n+2} \times 1$ using the arc which has defined the interior connected sum of $N_{j}^{\prime}$ and $N_{j}$. We call the result $I M_{j}^{\prime \prime}$ and then $\partial I M_{j}^{\prime \prime}=M_{j}^{\prime \prime} \cup N_{j}^{\prime \prime}$. By pushing the interiors of thickenings of arcs used for 
the boundary connected sum, into $S^{n+2} \times I$ and smoothing the corners, we have a boundary cobordism $I M_{1} \cup \cdots \cup I M_{j}^{\prime \prime} \cup \cdots \cup I M_{m}$, which will be denoted by $\alpha_{i j} \cdot I M$, between $\alpha_{i j} \cdot M$ and $\alpha_{i j} \cdot N$.

The following concepts are due to Cappell and Shaneson $[\mathrm{CS}]$. An $F_{m^{-}}$-link of dimension $n$ is defined as a pair $(L, \theta)$ where $L$ is a boundary $m$-link in $S^{n+2}$ and $\theta$ is a splitting map for $L$.

We fix some notations. Let $L_{i}$ be links with exteriors $X_{i}$ for $i=0,1$. Let $I L$ be a link cobordism between them. Then we let $I X$ be an exterior of $I L$ extending $X_{0}$ and $X_{1}$.

$F_{m}$-links $\left(L_{i}, \theta_{i}\right), i=0,1$, of dimension $n$ are $F_{m}$-cobordant if there is a link cobordism $I L$ in $S^{n+2} \times I$ of $L_{0}$ to $L_{1}$ and there is an epimorphism $\Theta: \Pi_{1} I X \rightarrow F_{m}$ such that $\theta_{i}=\Theta \cdot j_{i}$ up to inner automorphisms of $F_{m}$ for $i=0,1$ where $j_{i}: \Pi_{1} X_{i} \rightarrow$ $\Pi_{1} I X$ are induced from inclusions.

REMARK 2.5. Suppose that $f, g: A \rightarrow B$ are epimorphisms of groups. Then $f$ and $g$ differ by an inner automorphism of $A$ if and only if they differ by an inner automorphism of $B$. Thus, in the above definition of $F_{m}$-cobordism, the ambiguity in the choice of base points of $X_{0}, X_{1}$ and $I X$ is absorbed by inner automorphisms of $F_{m}$.

Such a pair $(I L, \Theta)$ is called an $F_{m^{-}}$cobordism of $\left(L_{0}, \theta_{0}\right)$ to $\left(L_{1}, \theta_{1}\right) . C_{n}\left(F_{m}\right)$ is defined to be the set of $F_{m}$-cobordism classes of $F_{m}$-links of dimension $n$.

$C A_{m}$ acts on $F_{m}$-links by composition, i.e., $\alpha \cdot(L \theta)=(L, \alpha \theta)$. This action naturally induces a well-defined action of $C A_{m}$ on $C_{n}\left(F_{m}\right)$ because $(I L, \alpha \Theta)$ is an $F_{m}$-cobordism of $\left(L_{i}, \alpha \theta_{i}\right), i=0,1$ for any $\alpha \in C A_{m}$ when $(I L, \Theta)$ is an $F_{m^{-}}$ cobordism of $\left(L_{i}, \theta_{i}\right), i=0,1$. Also we note that inner automorphisms of $F_{m}$ act trivially on $C_{n}\left(F_{m}\right)$ by the definition.

The following lemma is the link-cobordism version of Proposition 1.1.

Lemma 2.6. Let IL be a link cobordism of boundary links $L_{i}, i=0,1$. Then the following are equivalent:

(i) $I L$ is a boundary link cobordism for some choices of Seifert surfaces of $L_{0}$ to $L_{1}$.

(ii) There is an epimorphism $\Theta: \Pi_{1} I X \rightarrow F_{m}$ such that $\Theta \cdot j_{i}=\theta_{i}$ up to inner automorphisms of $F_{m}$ for some splitting maps $\theta_{i}$ of $L_{i}, i=0,1$.

PROOF. The same argument as in the proof of 1.1 works if we keep Remark 2.5 in mind. In addition, when we prove (ii) implies (i) we need the fact that two realizations $f_{1}, f_{1}^{\prime}: X_{1} \rightarrow \bigvee S^{1}$ of splitting maps $\theta_{1}, \theta_{1}^{\prime}: \Pi_{1} X_{1} \rightarrow F_{m}$, respectively, are homotopic if and only if $\theta_{1}=i \theta_{1}^{\prime}$ for some inner automorphism $i$ of $F_{m}[\mathbf{H u}$ ]. We note that the choice of Seifert surface of $L_{i}$ corresponds to the choice of splitting map for $L_{i}$, and vice versa, for each $i=0,1$.

Let $A_{m}$ be the quotient group of $C A_{m}$ by inner automorphisms of $F_{m}$. Applying Lemma 2.6 to the link cobordism which is a product, we see that inner automorphisms act trivially on $C_{n}\left(B_{m}\right)$. So we have the induced action of $A_{m}$ on $C_{n}\left(B_{m}\right)$.

Now we are ready to see:

THEOREM 2.7. There is a 1:1 correspondence between $C_{n}\left(B_{m}\right)$ and $C_{n}\left(F_{m}\right)$ which preserves the action of $A_{m}$. 
Proof. Proposition 2.1 gives us a map: $C_{n}\left(B_{m}\right) \rightarrow C_{n}\left(F_{m}\right)$ and Lemma 2.2 gives its inverse. The well-definedness of these maps is insured by Lemma 2.6. It remains to show that this map is equivariant under the action of $A_{m}$. Using the notations in the proof of 2.1, the map: $C_{n}\left(B_{m}\right) \rightarrow C_{n}\left(F_{m}\right)$ is given by $[M] \rightarrow\left[\left(\partial M, f_{*}\right)\right]$. We had the embedding $i: A \rightarrow X$ such that each arc of $i(A)$ did not intersect $M$. It is easy to see that only the $i$ th arc of $i(A)$ intersects the $j$ th surfaces of $\alpha_{i j} \cdot M$ and they intersect in only one point. Considering the positive normal direction, we can see that $\left[\alpha_{i j} \cdot M\right]$ goes to $\left[\left(\partial M, \alpha_{i j} f_{*}\right)\right]$ under this map.

We are going to make $C_{n}\left(B_{m}\right)$, and so $C_{n}\left(F_{m}\right)$, into an abelian group for $n \geq 2$. The second lemma of the next two lemmas is essential to do this. The other will be used in $\S 3$.

Lemma 2.8. Suppose $n \geq 3$. Then the following two equivalent statements hold.

(1) Every class of $C_{n}\left(F_{m}\right)$ contains a representative $(L, \theta)$ such that

(i) $\theta: \Pi_{1} X \rightarrow F_{m}$ is an isomorphism;

(ii) $\Pi_{i} X=0$ for $2 \leq i \leq[(n-1) / 2]$.

(2) Every class of $C_{n}\left(B_{m}\right)$ contains a representative $M=M_{1} \cup \cdots \cup M_{m}$ such that each $M_{i}$ is $[(n-1) / 2]$-connected.

Such a link $L$ or $\partial M$ as in Lemma 2.8 is called a simple link because the connectivity on complement or Seifert surface of a link in Lemma 2.8 is the strongest we can ask without making $L$ trivial. If $\theta$ is an isomorphism and $\Pi_{i} X=0$ for $2 \leq i \leq[(n+1) / 2]$ then $L$ is a trivial link for $n \geq 3$ [Gu, $\mathbf{C S}]$.

Before giving a proof, we observe the following:

SUBlemma 2.9. Let $X_{0}$ be an exterior of an m-link $L_{0}$ of dimension $n \geq 2$. And suppose that there is a smooth oriented manifold IX such that

(i) $\partial I X=X_{0} \cup X_{0} \times I \cup X_{1}$, so $\partial X_{1}=\partial X_{0}$;

(ii) $\Pi_{1} I X$ is normally generated by the images of a set of meridians of $\Pi_{1} X_{0}$ under the map induced by the inclusion;

(iii) IX is a homology cobordism of $X_{0}$ to $X_{1}$, that is, the inclusion: $X_{0} \rightarrow I X$ induces isomorphisms on all integral homology groups (by Poincaré duality, so does the inclusion: $\left.X_{1} \rightarrow I X\right)$.

Then $L_{0}$ is cobordant to a link whose exterior is diffeomorphic to $X_{1}$. Moreover, if there is an epimorphism $\Theta: \Pi_{1} I X \rightarrow F_{m}$ which restricts to a splitting map $\theta_{0}$ for $L_{0}$, then $\left(L_{0}, \theta_{0}\right)$ is $F_{m}$-cobordant to an $F_{m}$-link whose exterior is diffeomorphic to $X_{1}$.

Proof. Consider $U=I X \cup(T \times I)$ where $T$ is the tubular neighborhood of $L_{0}$ with $X_{0} \cup T=S^{n+2}$. By Van Kampen's theorem and condition (ii), $\Pi_{1} U$ is trivial. By Meyer-Vietoris sequences for $X_{0} \cup T$ and $I X \cup(T \times I)$, and condition (iii), and the five lemma, we have $H_{*} U=H_{*} S^{n+2}$. Thus $U$ is an $h$-cobordism of $S^{n+2}$. By the $h$-cobordism theorem (for the case $n=2$, [Sh]) there is a diffeomorphism $f: U \rightarrow S^{n+2} \times I$ fixing $X_{0} \cup(T \times 0)$. Since any diffeomorphism of $S^{n+2}$ is isotopic to the identity in the complement of an $(n+2)$-cell by the uniqueness of smooth disks, $f\left(L_{0} \times I\right)$ is a link cobordism of $L_{0}$ to a link $f\left(L_{0} \times 1\right)$ which is ambient isotopic to the link $L_{0} \times 1$ in $X_{1} \cup(T \times 1)$. 
The restriction $\theta_{1}: \Pi_{1} X_{1} \rightarrow F_{m}$ of $\Theta$ is a splitting map for the link $L_{0} \times 1$ in $X_{1} \cup T \times 1$. The link $F\left(L_{0} \times 1\right)$ has the splitting map carried by the ambient isotopy from $\theta_{1}$.

PROOF OF 2.8. Let $\left(L_{0}, \theta_{0}\right)$ be an $F_{m}$-link and $X_{0}$ an exterior of $L_{0}$. We want to do below-middle dimensional surgeries on the interior of $X_{0}$ to get a trace of surgeries $I X_{k}$ with the property $(*)$ :

(i) Let $\partial I X_{k}=X_{0} \cup \partial X_{0} \times I \cup X_{k}$. Then $\left(I X_{k}, X_{k}\right)$ is $(k+1)$-connected;

(ii) There is an isomorphism $\Theta_{k}: \Pi_{1}\left(I X_{k}\right) \rightarrow F_{m}$ which restricts to $\theta_{0}$;

(iii) $\Pi_{i} I X_{k}=0$ for $2 \leq i \leq k, k \leq[(n-1) / 2]$;

(iv) $I X_{k}$ is a homology cobordism.

Since $X_{0}$ is parallelizable and we do below-middle dimensional surgeries which can be framed, we can assume that all manifolds involved are stably parallelizable and all surgeries are framed.

Since $\theta_{0}: \Pi_{1} X_{0} \rightarrow F_{m}$ is onto, according to $[\mathbf{C a}] \operatorname{ker}\left(\theta_{0}\right)$ is normally generated by $\alpha_{1}, \ldots, \alpha_{l}$ for some $\alpha_{i} \in \Pi_{1} X_{0}$. We kill $\alpha_{1}, \ldots, \alpha_{l}$ by surgery to have $I X_{1}^{\prime}=$ $X_{0} \times I \cup 2$-handles along $\alpha_{1}, \ldots, \alpha_{l}$. Then $\Theta_{1}^{\prime}: \Pi_{1}\left(I X_{1}^{\prime}\right) \rightarrow F_{m}$ induced by $\theta_{0}$, is now an isomorphism and $\left(I X_{1}^{\prime}, X_{1}^{\prime}\right)$ is $n$-connected where $X_{1}^{\prime}=\partial I X_{1}^{\prime}-X_{0}$. Since $\alpha_{1}, \ldots, \alpha_{l}$ are null homologous, Mayer-Vietoris sequence shows that $H_{2} I X_{1}^{\prime}$ is a free abelian group with $l$ generators and $H_{i} I X_{1}^{\prime} \cong H_{i} X_{0}$ for $i \geq 3$. The Hopf sequence $\Pi_{2} I X_{1}^{\prime} \rightarrow H_{2} I X_{1}^{\prime} \rightarrow H_{2} F_{m} \rightarrow 0$ implies that $H_{2} I X_{1}^{\prime}$ is all spherical. Thus we can kill $H_{2} I X_{1}^{\prime}$ by adding 3 -handles to get $I X_{1}$ without introducing any new homology. Then $\Pi_{1} I X_{1}^{\prime} \cong \Pi_{1} I X_{1}$ and $\left(I X_{1}, X_{1}\right)$ is $(n-1)$-connected. Since $n \geq 3$, we have $I X_{1}$ satisfying $(*)$.

Now we do an induction on $k$. Assume that we have $I X_{k-1}$ satisfying $(*)$. $\Pi_{k} X_{k-1}$ is generated by relative $k$-cells in the pair $\left(U, \bigvee S^{1}\right)$ as a $\mathbf{Z}\left[F_{m}\right]$-module where $U$ is the mapping cylinder of a map: $X_{k-1} \rightarrow \bigvee S^{1}$ realizing $\theta_{k-1}$. Thus $\Pi_{k} I X_{k-1} \cong \Pi_{k} X_{k-1}$ is finitely generated as a $\mathbf{Z}\left[F_{m}\right]$-module. We kill $\Pi_{k} X_{k-1}$ by adding $(k+1)$-handles to $I X_{k-1}$ to get $I X_{k}^{\prime}$. The Mayer-Vietoris sequence shows that $H_{k+1} I X_{k}^{\prime}$ is a finitely generated free abelian group and $H_{i} I X_{k}^{\prime} \cong H_{i} I X_{k-1} \cong$ $H_{i} X_{0}$ for $i \neq k+1$. The Cartan-Leray spectral sequence [Mc] of the universal covering of $I X_{k}^{\prime}$ gives the exact sequence

$$
\Pi_{k+1} I X_{k}^{\prime} \rightarrow H_{k+1} I X_{k}^{\prime} \rightarrow H_{k+1} F_{m} \rightarrow 0 .
$$

Thus $H_{k+1} I X_{k}^{\prime}$ is all spherical and so we can kill $H_{k+1} I X_{k}^{\prime}$ by adding $(k+2)$-handles to $I X_{k}^{\prime}$ to get $I X_{k}$. Since $H_{k+1} I X_{k}^{\prime}$ was free, no new homology is introduced on $I X_{k}$. Since $\left(I X_{k}^{\prime}, I X_{k-1}\right)$ is $k$-connected and $\left(I X_{k}, I X_{k}^{\prime}\right)$ is $(k+1)$-connected, $\Theta_{k}$ is an isomorphism induced from $\Theta_{k-1}$ and $\Pi_{i} I X_{k}=0$ for $2 \leq i \leq k$. And since $\left(I X_{k}, X_{k}\right)$ is $(n-k)$-connected and $k \leq[(n-1) / 2]$, it is $(k+1)$-connected. We also see that $H_{*} I X_{k} \cong H_{*} X_{0}$. Part (1) of 2.8 follows from putting $k=[(n-1) / 2]$ and Sublemma 2.9.

Theorem 2.7 and the unlinking result in $[\mathbf{G u}]$ show that (1) implies (2) of 2.8. For such a representative $F_{m}$-link $(L, \theta)$, let $f: X \rightarrow \bigvee S^{1}$ be a realization of $\theta$ and let $M=M_{1} \cup \cdots \cup M_{m}$ be a Seifert surface obtained from the transversality of $f$. The unlinking theorem $[\mathbf{G u}]$ says that if $f$ is $(k+1)$-connected then one can do ambient surgeries on $M$ to obtain a new Seifert surface $N=N_{1} \cup \cdots \cup N_{m}$ with each $N_{i}$ being $k$-connected. Then $M$ and $N$ represent the same class in $C_{n}\left(B_{m}\right)$ because they are cobordant relative boundary. 
The implication from (2) to (1) can be obtained from the construction of the universal covering of an exterior of a given simple link by cutting and gluing through the $[(n-1) / 2]$-connected Seifert surface $[\mathbf{G u 1}]$.

LEMMA 2.10. If $n \geq 2$, the following equivalent statements hold:

(1) If two $F_{m}$-links of dimension $n$ are $F_{m}$-cobordant then there is an $F_{m}$ cobordism $(I L, \Theta)$ of them such that $\Theta$ is an isomorphism.

(2) If two Seifert surfaces are boundary cobordant then there is a boundary cobordism IM of them such that each component of IM is 1-connected.

Proof. (1) $[\mathbf{C S}]$ Let $\left(I L^{\prime}, \Theta^{\prime}\right)$ be an $F_{m}$-cobordism with an exterior $I X^{\prime}$. We do surgeries of index 2 on the interior of $I X^{\prime}$ to kill $\operatorname{ker} \Theta^{\prime}$. And we do surgeries of index 3 to kill the integral homology classes produced by the previous surgeries. By Sublemma 2.9 we have an $F_{m}$-cobordism, whose splitting map is an isomorphism, of $F_{m}$-links which are ambient isotopic to the given $F_{m}$-links. By adding the ambient isotopy to the $F_{m}$-cobordism, we get a desired $F_{m}$-cobordism.

To show that (1) implies (2), let $I M=I M_{1} \cup \cdots \cup I M_{m}$ be any Seifert surface of the $F_{m}$-cobordism $(I L, \Theta)$ as in Lemma 2.6. Let $\widetilde{I X}$ be the universal cover of $I X$. Then $\widetilde{I X}$ can be constructed by cutting and pasting $I X$ along $I M$ as usual. Thus the map: $\Pi_{1} I M \rightarrow \Pi_{1} I X$ induced from the inclusion factors through $\Pi_{1} \widetilde{I X}$ which is the trivial group. Thus the map: $\Pi_{1} I M_{k} \rightarrow \Pi_{1} I X$ induced from the inclusion is zero for each $k=1, \ldots, m$.

We define a function $N$ from the set of Seifert surfaces of $(I L, \Theta)$ to the set of nonnegative integers as in $[\mathbf{L} \mathbf{1}]$. For a set $\left\{\alpha_{i}\right\}$ of generators for $\Pi_{1} I M$ and a set of embeddings $\left\{f_{i}:\left(D^{2}, \partial D^{2}\right) \rightarrow(\operatorname{int} I X\right.$, int $I M)$ such that $f_{i}$ is transverse to $I M$ and $f_{i}\left(\partial D^{2}\right)$ represent $\left.\alpha_{i}\right\}$, we define $N\left(\left\{\alpha_{i}\right\},\left\{f_{i}\right\}\right)=\sum_{i}$ order of $\Pi_{0}\left(f_{i}\left(D^{2}\right) \cap I M\right)$ and $N(I M)$ to be the minimum of $N\left(\left\{\alpha_{i}\right\},\left\{f_{i}\right\}\right)$ for all choices of $\left\{\alpha_{i}\right\}$ and $\left\{f_{i}\right\}$. It is easy to see that $N(I M)=0$ if and only if each component of $I M$ is 1 connected. By induction, we will be done if we can find a new Seifert surface $I N$ of $(I L, \Theta)$ with $N(I N)<N(I M)$ for $I M$ with $N(I M)>0$. Let $\left\{\alpha_{i}\right\},\left\{f_{i}\right\}$ be the sets defined as above for $I M$ such that $N(I M)=N\left(\left\{\alpha_{i}\right\},\left\{f_{i}\right\}\right)$. The innermost component of $f_{1}\left(D^{2}\right) \cap I M$ can not be null homotopic in $I M$, for if it were then $f_{1}$ could be replaced by another embedding $f_{1}^{\prime}$ with the same property except one less component of $f_{1}^{\prime}\left(D^{2}\right) \cap I M$ using a disk in $I M$ bounded by the innermost circle. Then we do a surgery of index 2 on $I M$ using the disk in $f_{1}\left(D^{2}\right)$ bounded by the innermost circle as the core of a 2-handle. We may assume that the 2-handle does not intersect $I M$. The new Seifert surface $I N$ obtained from this surgery obviously has the property that $N(I N)<N(I M)$.

The implication of (1) from (2) follows from the construction of $\widetilde{I X}$ using $I M$ if we show that $S^{n+2} \times I-I M$ is also 1-connected. Let

$$
Y_{k}=S^{n+2} \times I-\left(I M_{1} \cup \cdots \cup I M_{k}\right)
$$

for $k=1, \ldots, m$ where $I M=I M_{1} \cup \cdots \cup I M_{m}$. By induction it suffices to show $Y_{k}$ is 1-connected assuming that $Y_{k-1}$ is 1-connected. Let $R$ be a closed regular neighborhood of $I M_{k}$ in $S^{n+2} \times I$. Then $Y_{k}$ is homotopy equivalent to the closure $C R$ of $Y_{k-1}-R$. But $C R \cap R$ is homotopy equivalent to $I M_{k} \cup_{S} I M_{k}$ where $S$ is the middle part of $\partial I M_{k}$ which is homeomorphic to $S^{n} \times I$. Since $n \geq 2$ and $I M_{k}$ 
is 1-connected, $\Pi_{1}\left(I M_{k} \cup_{S} I M_{k}\right)$ is trivial and so $\Pi_{1}(C R \cap R)$ is trivial. Since $\Pi_{1} R$ is trivial, $\Pi_{1} C R$ is trivial by Van Kampen's theorem. Thus $\Pi_{1} Y_{k}$ is trivial.

We now define an addition + on $C_{n}\left(B_{m}\right)$ for $n \geq 2$. Let $M=M_{1} \cup \cdots \cup M_{m}$ and $N=N_{1} \cup \cdots \cup N_{m}$ be in $S(n, m)$. Assume that $M$ and $N$ are embedded into two hemispheres separately in $S^{n+2}$. We choose disjoint $\operatorname{arcs} \gamma_{i}$ for $i=1, \ldots, m$ joining a point in $\partial M_{i}$ and a point in $\partial N_{i}$ and being away from $M$ and $N$ except their ends. Such arcs exist because $M \cup N$ has no closed components. $M_{i} \natural_{\gamma_{i}} N_{i}$ denotes the boundary connected sum of $M_{i}$ and $N_{i}$ along $\gamma_{i}$. We put

$$
M \natural_{\left(\gamma_{1}, \ldots, \gamma_{m}\right)} N=\left(M_{1} \natural_{\gamma_{1}} N_{1}\right) \cup \cdots \cup\left(M_{m} \natural_{\gamma_{m}} N_{m}\right) \text {. }
$$

Note that this boundary connected sum does not depend on thickenings of $\gamma_{i}$ 's up to ambient isotopy for $n \geq 2$. Let $[M]$ and $[N]$ be the classes of $M$ and $N$ in $C_{n}\left(B_{m}\right)$. We define $[M]+[N]$ by $\left[M \natural_{\left(\gamma_{1}, \ldots, \gamma_{m}\right)} N\right]$.

Proposition 2.11. + is well defined on $C_{n}\left(B_{m}\right)$ for $n \geq 2$.

Proof. We have to show that if $M$ and $N$ are boundary cobordant to $M^{\prime}$ and $N^{\prime}$ by $I M$ and $I N$, respectively, and $\left(\gamma_{1}, \ldots, \gamma_{m}\right)$ and $\left(\gamma_{1}^{\prime}, \ldots, \gamma_{m}^{\prime}\right)$ are sets of arcs as above joining $M$ and $N$ and joining $M^{\prime}$ and $N^{\prime}$, respectively, then

$$
M \natural_{\left(\gamma_{1}, \ldots, \gamma_{m}\right)} N \text { is boundary cobordant to } M^{\prime} \natural_{\left(\gamma_{1}^{\prime}, \ldots, \gamma_{m}^{\prime}\right)} N^{\prime} \text {. }
$$

By Lemma 2.10 we may assume that each component of $I M$ and $I N$ is 1-connected. We may also assume that $I M$ and $I N$ are embedded disjointly in $S^{n+2} \times I$. Arguing as in the proof of 2.10, the complement of $I M \cup I N$ in $S^{n+2} \times I$ is 1-connected.

We choose $\operatorname{arcs} \sigma_{i}$ for $i=1, \ldots, m$ in $\partial I M_{i}-\operatorname{int}\left(M_{i} \cup M_{i}^{\prime}\right)$ joining the starting points of $\gamma_{i}$ and $\gamma_{i}^{\prime}$ and $\operatorname{arcs} \tau_{i}$ for $i=1, \ldots, m$ in $\partial I N_{i}-\operatorname{int}\left(N_{i} \cup N_{i}^{\prime}\right)$ joining the end points of $\gamma_{i}^{\prime}$ and $\gamma_{i}$. Since $S^{n+2} \times I-(I M \cup I N)$ is 1-connected, the simple closed loops $\sigma_{i} \gamma_{i}^{\prime} \tau_{i} \gamma_{i}^{-1}$ bound disjoint smooth 2-disks $\Delta_{i}$ in $S^{n+2} \times I$ for $n \geq 2$ such that int $\Delta_{i}$ does not intersect $I M \cup I N$. We choose closed tubular neighborhoods $T_{i}$ for $i=1, \ldots, m$ of $\Delta_{i}$ in $S^{n+2} \times I$ such that

(i) $I M \cap T_{i}$ and $I N \cap T_{i}$ are homeomorphic to $D^{n} \times I$;

(ii) $S^{n+2} \times 0 \cap T_{i}$ and $S^{n+2} \times 1 \cap T_{i}$ are tubular neighborhoods of $\gamma_{i}$ and $\gamma_{i}^{\prime}$, respectively.

There is a given normal 1-frame on $\partial \Delta_{i}$ in $S^{n+2} \times I$, that is, on $\sigma_{i}$ and $\tau_{i}$ given by the positive normal directions of $I M$ and $I N$ in $S^{n+2} \times I$, and on $\gamma_{i}$ and $\gamma_{i}^{\prime}$ given by the constructions of the boundary connected sums $M \natural_{\left(\gamma_{1}, \ldots, \gamma_{m}\right)} N$ and $M^{\prime} \natural_{\left(\gamma_{1}^{\prime}, \ldots, \gamma_{m}^{\prime}\right)} N^{\prime}$. We extend this normal 1 -frame on $\partial \Delta_{i}$ to $\Delta_{i}$ for each $i$. This is possible because $G_{n+1,1}^{+}$, the space of oriented lines in $\mathbf{R}^{n+1}$, is 1-connected for $n \geq 2$. Let $b_{i}, i=1, \ldots, m$ be the thickenings of $\Delta_{i}$ orthogonal to these 1 -frames in $T_{i}$. We put

$$
I M \natural_{\left(\Delta_{1}, \ldots, \Delta_{m}\right)} I N=\left(I M_{1} \cup b_{1} \cup I N_{1}\right) \cup \cdots \cup\left(I M_{m} \cup b_{m} \cup I N_{m}\right)
$$

after smoothing the corners. Clearly $M \natural_{\left(\gamma_{1}, \ldots, \gamma_{m}\right)} N$ is boundary cobordant to $M^{\prime} \natural_{\left(\gamma_{1}^{\prime}, \ldots, \gamma_{m}^{\prime}\right)} N^{\prime}$ by $I M \natural_{\left(\Delta_{1}, \ldots, \Delta_{m}\right)} I N$.

Let $E$ be $m$ copies of $D^{n+1}$ disjointedly embedded in $S^{n+2}$. Any choice of orientations for $E$ gives the same class in $C_{n}\left(B_{m}\right)$. It is easy to see that $[E]$ is the identity element in $C_{n}\left(B_{m}\right)$. 
REMARK 2.12. Let $M \in S(n, m)$. Then $M$ is boundary cobordant to $E$ if and only if there is a submanifold $V^{n+2}$ in the disk $D^{n+3}$ bounded by the ambient sphere $S^{n+2}$ such that

(i) $V \cap S^{n+2}=\partial V \cap S^{n+2}=M$;

(ii) $\partial V-\operatorname{int} M$ is homeomorphic to $m$ copies of $D^{n+1}$;

(iii) $V$ is a disjoint ordered union of oriented submanifolds $V_{1}, \ldots, V_{m}$ of $D^{n+3}$ with the positive normal direction which restricts to the positive normal directions of $M$ on $\partial D^{n+3}$.

In this situation we say that the boundary $m$-link $\partial M$ is boundary slice.

As in the case of knots, the inverse of an element $[M]$ of $C_{n}\left(B_{m}\right)$ is constructed as follows: We assume that $M$ is embedded in a hemisphere of $S^{n+2}$. Then $-M$ is defined to be the image of $M$ with the reversed orientation under the reflection of $S^{n+2}$ leaving the boundary of the hemisphere fixed. It is straightforward to prove that $[M]+[-M]=[E]$ using a spinning argument in which we should choose a special set of arcs to define a boundary connected sum. Consequently, $C_{n}\left(B_{m}\right)$ is an abelian group under + for $n \geq 2$. By Theorem 2.7 this abelian group structure is carried over to $C_{n}\left(F_{m}\right)$.

3. Seifert matrices and boundry link cobordisms. In this section we will discuss an algebraic gadget called a Seifert matrix which is associated to a Seifert surface in $S(2 q-1, m)$ and we will identify $C_{2 q-1}\left(B_{m}\right)$ with a set of Seifert matrices modulo a relation called cobordism.

Meanwhile, any even dimensional boundary $m$-link is known to be boundary sliced with any choice of Seifert surface of the link [Ke and Gu1]. This is essentially due to the fact that the odd dimensional surgery obstruction group of the trivial group is trivial. Thus $C_{2 q}\left(B_{m}\right)=C_{2 q}\left(F_{m}\right)=0$ for all $q$.

All matrices in this section will have integer entries. A matrix $A=\left(A_{i j}\right)_{i, j=1, \ldots, m}$ of $m^{2}$ matrices is called a Seifert matrix of type $(m, \varepsilon)$ for $\varepsilon= \pm 1$ if

(i) for $i=1, \ldots, m A_{i i}$ is a $\left(2 l_{i} \times 2 l_{i}\right)$-matrix with the property that $A_{i i}+\varepsilon A_{i i}^{T}$ is unimodular;

(ii) for $i, j=1, \ldots, m$ and $i \neq j A_{i j}$ is a $\left(2 l_{i} \times 2 l_{j}\right)$-matrix with the property that $A_{i j}=-\varepsilon A_{j i}^{T}$ where $l_{i}$ are nonnegative integers and $T$ stands for the transpose of matrices.

We note that $A+\varepsilon A^{T}$ is unimodular if and only if $A_{i i}+\varepsilon A_{i i}^{T}$ are unimodular for all $i=1, \ldots, m$.

Let $A=\left(A_{i j}\right)$ and $B=\left(B_{i j}\right)$ be Seifert matrices of type $(m, \varepsilon) . A$ is said to be congruent ${ }^{3}$ to $B$ if there are unimodular matrices $U_{i}$ for $i=1, \ldots, m$ such that $U_{i} A_{i j} U_{j}^{T}=B_{i j}$ for all $i, j$. The block sum $A \oplus B$ is defined to be $\left(A_{i j} \oplus B_{i j}\right)$ where

$$
A_{i j} \oplus B_{i j}=\left(\begin{array}{cc}
A_{i j} & 0 \\
0 & B_{i j}
\end{array}\right) .
$$

$A \oplus B$ is obviously a Seifert matrix of type $(m, \varepsilon)$ again.

A Seifert matrix of type $(m, \varepsilon)$ is said to be null cobordant if it is congruent to a Seifert matrix $N=\left(N_{i j}\right)$ of the same type such that for each $i, j, N_{i j}$ has the form

$$
\left(\begin{array}{cc}
0 & C_{i j} \\
D_{i j} & E_{i j}
\end{array}\right)
$$

${ }^{3}$ One should distinguish this from the usual congruence. 
where $C_{i j}, D_{i j}$ and $E_{i j}$ are $\left(l_{i} \times l_{j}\right)$-matrices and $N_{i j}$ is a $\left(2 l_{i} \times 2 l_{j}\right)$-matrix. We say that Seifert matrices $A$ and $B$ of the same type are cobordant if $A \oplus(-B)$ is null cobordant.

Lemma 3.1. If $A$ and $N$ are Seifert matrices of type $(m, \varepsilon)$ with $N$ and $A \oplus N$ being null cobordant, then $A$ is null cobordant.

Proof. Let $A=\left(A_{i j}\right)$ and $N=\left(N_{i j}\right)$. We consider $A_{i j}$ and $N_{i j}$ as bilinear forms:

$$
A_{i j}: A_{i} \times A_{j} \rightarrow \mathbf{Z}, \quad N_{i j}: N_{i} \times N_{j} \rightarrow \mathbf{Z}
$$

and so

$$
A_{i j} \oplus N_{i j}:\left(A_{i} \oplus N_{i}\right) \times\left(A_{j} \oplus N_{j}\right) \rightarrow \mathbf{Z}
$$

where $A_{i}, N_{i}, i=1, \ldots, m$, are free abelian groups with even ranks. By the hypothesis there are subgroups $K_{i}$ of $A_{i} \oplus N_{i}$ and $N_{i}^{\prime}$ of $N_{i}$ for $i=1, \ldots, m$ such that for all $i, j$

(i) $\left(A_{i j} \oplus N_{i j}\right)\left(K_{i}, K_{j}\right)=0$;

(ii) $N_{i j}\left(N_{i}^{\prime}, N_{j}^{\prime}\right)=0$

(iii) $\operatorname{rk}\left(K_{i}\right)=\frac{1}{2} \operatorname{rk}\left(A_{i} \oplus N_{i}\right)$ and $\operatorname{rk}\left(N_{i}^{\prime}\right)=\frac{1}{2} \operatorname{rk}\left(N_{i}\right)$.

The following argument is applied for all $i, j=1, \ldots, m$ without saying it. Let $N_{i}^{\prime \prime}$ be a subgroup of $N_{i}$ such that $N_{i}=N_{i}^{\prime} \oplus N_{i}^{\prime \prime}$. Let $p_{i}, p_{i}^{\prime}$ and $p_{i}^{\prime \prime}$ be projections from $A_{i} \oplus N_{i}^{\prime} \oplus N_{i}^{\prime \prime}$ onto $A_{i}, N_{i}^{\prime}$ and $N_{i}^{\prime \prime}$, respectively. Let $K_{i}^{\prime}$ be a subgroup of $K_{i}$ defined to be $\left(\operatorname{ker} p_{i}^{\prime \prime}\right) \cap K_{i}$ or equivalently to be $\left(A_{i} \oplus N_{i}^{\prime}\right) \cap K_{i}$. Then we claim that $p_{i}\left(K_{i}^{\prime}\right)$ is the desired subgroup of $A_{i}$, that is, we must show

(i) $A_{i j}\left(p_{i}\left(K_{i}^{\prime}\right), p_{j}\left(K_{j}^{\prime}\right)\right)=0$;

(ii) $\operatorname{rk}\left(p_{i}\left(K_{i}^{\prime}\right)\right)=\frac{1}{2} \operatorname{rk}\left(A_{i}\right)$.

To see (i), let $k_{i} \in K_{i}^{\prime}$ and $k_{j} \in K_{j}^{\prime}$. Then

$$
\begin{aligned}
A_{i j}\left(p_{i}\left(k_{i}\right), p_{j}\left(k_{j}\right)\right) & =A_{i j}\left(p_{i}\left(k_{i}\right), p_{j}\left(k_{j}\right)\right)+N_{i j}\left(p_{i}^{\prime}\left(k_{i}\right), p_{j}^{\prime}\left(k_{j}\right)\right) \\
& =A_{i j}\left(p_{i}\left(k_{i}\right), p_{j}\left(k_{j}\right)\right)+N_{i j}\left(\left(p_{i}^{\prime} \oplus p_{i}^{\prime \prime}\right)\left(k_{i}\right),\left(p_{j}^{\prime} \oplus p_{j}^{\prime \prime}\right)\left(k_{j}\right)\right) \\
& =\left(A_{i j} \oplus N_{i j}\right)\left(k_{i}, k_{j}\right) \\
& =0 .
\end{aligned}
$$

To see (ii), let $K_{i}^{\prime \prime}=\left(\operatorname{ker} p_{i}\right) \cap K_{i}^{\prime}=N_{i}^{\prime} \cap K_{i}$. Then we claim that

$$
N_{i i}\left(p_{i}^{\prime}\left(K_{i}^{\prime \prime}\right), p_{i}^{\prime \prime}\left(K_{i}\right)\right)=0=N_{i i}\left(p_{i}^{\prime \prime}\left(K_{i}\right), p_{i}^{\prime}\left(K_{i}^{\prime \prime}\right)\right) .
$$

To show the claim, let $k_{i}^{\prime \prime} \in K_{i}^{\prime \prime}$ and $k_{i} \in K_{i}$. Then

$$
\begin{aligned}
0 & =\left(A_{i i} \oplus N_{i i}\right)\left(k_{i}^{\prime \prime}, k_{i}\right) \\
& =A_{i i}\left(p_{i}\left(k_{i}^{\prime \prime}\right), p_{i}\left(k_{i}\right)\right)+N_{i i}\left(p_{i}^{\prime}\left(k_{i}^{\prime \prime}\right)+p_{i}^{\prime \prime}\left(k_{i}^{\prime \prime}\right), p_{i}^{\prime}\left(k_{i}\right)+p_{i}^{\prime \prime}\left(k_{i}\right)\right) \\
& =N_{i i}\left(p_{i}^{\prime}\left(k_{i}^{\prime \prime}\right), p_{i}^{\prime}\left(k_{i}\right)+p_{i}^{\prime \prime}\left(k_{i}\right)\right) \\
& =N_{i i}\left(p_{i}^{\prime}\left(k_{i}^{\prime \prime}\right), p_{i}^{\prime \prime}\left(k_{i}\right)\right) .
\end{aligned}
$$

Similarly $0=N_{i i}\left(p_{i}^{\prime \prime}\left(k_{i}\right), p_{i}^{\prime}\left(k_{i}^{\prime \prime}\right)\right)$. Thus the subgroup $p_{i}^{\prime}\left(K_{i}^{\prime \prime}\right)$ of $N_{i}^{\prime}$ is orthogonal to the subgroup $p_{i}^{\prime \prime}\left(K_{i}\right)$ of $N_{i}^{\prime \prime}$ with respect to a unimodular form $N_{i i}+\varepsilon N_{i i}^{T}$ which is a nonsingular pairing of $N_{i}^{\prime}$ with $N_{i}^{\prime \prime}$. Thus $\operatorname{rk}\left(p_{i}^{\prime}\left(K_{i}^{\prime \prime}\right)\right)+\operatorname{rk}\left(p_{i}^{\prime \prime}\left(K_{i}\right)\right) \leq \operatorname{rk}\left(N_{i}^{\prime}\right)$. Since $p_{i}^{\prime}\left(K_{i}^{\prime \prime}\right) \cong K_{i}^{\prime \prime}$,

$$
\operatorname{rk}\left(K_{i}^{\prime \prime}\right)+\operatorname{rk}\left(p_{i}^{\prime \prime}\left(K_{i}\right)\right) \leq \operatorname{rk}\left(N_{i}^{\prime}\right) \cdots(*) .
$$


The exact sequences

$$
0 \rightarrow K_{i}^{\prime} \rightarrow K_{i} \stackrel{p_{i}^{\prime \prime}}{\rightarrow} p_{i}^{\prime \prime}\left(K_{i}\right) \rightarrow 0
$$

and

$$
0 \rightarrow K_{i}^{\prime \prime} \rightarrow K_{i}^{\prime} \stackrel{p_{i}}{\rightarrow} p_{i}\left(K_{i}^{\prime}\right) \rightarrow 0
$$

give that $\operatorname{rk}\left(p_{i}^{\prime \prime}\left(K_{i}\right)\right)=\operatorname{rk}\left(K_{i}\right)-\operatorname{rk}\left(K_{i}^{\prime \prime}\right)-\operatorname{rk}\left(p_{i}\left(K_{i}^{\prime}\right)\right)$. By substituting this in (*), we have $\operatorname{rk}\left(K_{i}\right)-\operatorname{rk}\left(p_{i}\left(K_{i}^{\prime}\right)\right) \leq \operatorname{rk}\left(N_{i}^{\prime}\right)$. Thus, $\operatorname{rk}\left(p_{i}\left(K_{i}^{\prime}\right)\right) \geq \operatorname{rk}\left(K_{i}\right)-\operatorname{rk}\left(N_{i}^{\prime}\right)=$ $\frac{1}{2} \operatorname{rk}\left(A_{i}\right)$. Combining this with the fact that $A_{i i}+\varepsilon A_{i i}^{T}$ is unimodular, we have $\operatorname{rk}\left(p_{i}\left(K_{i}^{\prime}\right)\right)=\frac{1}{2} \operatorname{rk}\left(A_{i}\right)$.

COROLlaRY 3.2. The cobordism on Seifert matrices of type $(m, \varepsilon)$ is an equivalence relation.

ProOF. One can check that the operation $\oplus$ is commutative and compatible with cobordism. One can also check that $A \oplus(-A)$ is null cobordant. Let $A, B$ and $C$ be Seifert matrices such that $A$ is cobordant to $B$ and $B$ is cobordant to $C$. Then $A \oplus(-B)$ and $B \oplus(-C)$ are null cobordant and hence $A \oplus(-B) \oplus B \oplus(-C)$ is null cobordant. Since $(-B) \oplus B$ is null cobordant, $A \oplus(-C)$ is null cobordant by Lemma 3.1 .

$G(m, \varepsilon)$ denotes the set of cobordism classes of Seifert matrices of type $(m, \varepsilon)$. Then $G(m, \varepsilon)$ is an abelian group under $\oplus$ because the null-cobordant class serves as the identity and the class of $-A$ is the inverse of the class of $A$.

We will construct a map

$$
\phi(m, q): C_{2 q-1}\left(B_{m}\right) \rightarrow G\left(m,(-1)^{q}\right) .
$$

But we first assign a Seifert matrix to each element of $S(2 q-1, m)$. Let $M=M_{1} \cup$ $\cdots \cup M_{m}$ be a Seifert surface in $S(2 q-1, m)$. The map $i_{+}: H_{*} M \rightarrow H_{*}\left(S^{2 q-1}-M\right)$ is induced from a slight translation: $M \rightarrow S^{2 q-1}-M$ along the positive normal direction of $M$ in the ambient sphere $S^{2 q-1}$. The Seifert pairing on $M$ is a bilinear map

$$
\sigma: H_{q} M \times H_{q} M \rightarrow \mathbf{Z}
$$

defined by $\sigma(\alpha, \beta)=\mathrm{lk}\left(\alpha, i_{+} \beta\right)$ for $\alpha, \beta \in H_{q} M$ where lk denotes the linking pairing in the ambient sphere. Then we have that

$$
\sigma(\alpha, \beta)+(-1)^{q} \sigma(\beta, \alpha)=\alpha \cdot \beta
$$

where $\alpha \cdot \beta$ is the intersection number of $\alpha$ and $\beta$ in $M$ (see [L2]). By choosing a basis for the torsion free part of $H_{q} M=H_{q} M_{1} \oplus \cdots \oplus H_{q} M_{m}$ in order, we have an associated matrix $A=\left(A_{i j}\right)$ for $\sigma$ where $A_{i j}$ is the associated matrix to the restriction of $\sigma$ to $H_{q} M_{i} \times H_{q} M_{j}$ for each $i, j=1, \ldots, m$. Since $A_{i i}+$ $(-1)^{q} A_{i i}^{T}$ represents the intersection pairing on $H_{q} M_{i}$ and the boundary of $M_{i}$ is homeomorphic to $S^{2 q-1}$, it is unimodular by Poincaré duality for each $i=1, \ldots, m$. And since $M_{i}$ and $M_{j}$ are disjoint, $A_{i j}+(-1)^{q} A_{j i}^{T}=0$ for all $i \neq j$. Thus the matrix $A$ is a Seifert matrix of type $\left(m,(-1)^{q}\right)$.

Let $A, B$ be Seifert matrices for Seifert surfaces $M, N$, respectively. Then it is clear that $A \oplus B$ is a Seifert matrix of the Seifert surface $M \natural N$ and the choice of arcs for boundary connected sums does not matter. It is also clear that $-A$ is a Seifert matrix of $-M$. 
LEMMA 3.3. Let $M$ be a Seifert surface in $S(2 q-1, m)$ and $A$ a Seifert matrix of $M$. If $M$ represents the identity class in $C_{2 q-1}\left(B_{m}\right)$, then $A$ is null cobordant.

PROOF. Let $V=V_{1} \cup \cdots \cup V_{m}$ be a submanifold in $D^{2 q+2}$ as in Remark 2.12. Let $j_{i}: H_{q} M_{i} \rightarrow H_{q} V_{i}, i=1, \ldots, m$, be homomorphisms induced from inclusions. Let $K_{i}$ be the kernel of $j_{i}$. Let $\alpha \in K_{i}$ and $\beta \in K_{j}$. Then $\alpha, \beta$ bound chains $c_{\alpha}, c_{\beta}$ in $V$. Let $\bar{i}_{+}: H_{q} V \rightarrow H_{q}\left(D^{2 q+2}-V\right)$ be the map induced from a slight translation along the positive normal direction on $V$. Then

$$
\sigma_{i j}(\alpha, \beta)=\operatorname{lk}\left(\alpha, i_{+} \beta\right)=c_{\alpha} \cdot\left(\bar{i}+c_{\beta}\right)=0 .
$$

To see that $\operatorname{rk}\left(K_{i}\right)=\frac{1}{2} \operatorname{rk}\left(H_{q} M_{i}\right)$, we consider the exact sequence

$$
H_{q+1} V_{i} \stackrel{\lambda}{\rightarrow} H_{q+1}\left(V_{i}, M_{i}\right) \stackrel{\partial}{\rightarrow} H_{q} M_{i} \stackrel{j_{i}}{\rightarrow} H_{q} V_{i} \stackrel{\eta}{\rightarrow} H_{q}\left(V_{i}, M_{i}\right) .
$$

Since $\partial V_{i}-\operatorname{int} M_{i}$ is homeomorphic to $D^{2 q}$, we have

$$
H_{q+1}\left(V_{i}, M_{i}\right) \cong H^{q}\left(V_{i}, D^{2 q}\right) \cong H^{q} V_{i}
$$

and

$$
H_{q}\left(V_{i}, M_{i}\right) \cong H^{q+1}\left(V_{i}, D^{2 q}\right) \cong H^{q+1} V_{i} .
$$

And modulo torsions, $H^{q} V_{i} \cong \operatorname{Hom}\left(H_{q} V_{i}, \mathbf{Z}\right)$ and $H^{q+1} V_{i} \cong \operatorname{Hom}\left(H_{q+1} V_{i}, \mathbf{Z}\right)$. Thus both homomorphisms $H_{q+1} V_{i} \rightarrow \operatorname{Hom}\left(H_{q} V_{i}, \mathbf{Z}\right)$ and $H_{q} V_{i} \rightarrow \operatorname{Hom}\left(H_{q+1} V_{i}, \mathbf{Z}\right)$ obtained from $\lambda$ and $\eta$, respectively, by composing with these isomorphisms, are adjoints of the intersection pairing $H_{q} V_{i} \times H_{q+1} V_{i} \rightarrow \mathbf{Z}$. In particular, $\operatorname{rk}(\operatorname{im} \lambda)=$ $\operatorname{rk}(\operatorname{im} \eta)$. By looking at the first three terms and the last three terms of the exact sequence, we have

$$
\operatorname{rk}(\operatorname{im} \lambda)+\operatorname{rk}(\operatorname{im} \partial)=\operatorname{rk}\left(H_{q+1}\left(V_{i}, M_{i}\right)\right)=\operatorname{rk}\left(H_{q} V_{i}\right)=\operatorname{rk}(\operatorname{im} \eta)+\operatorname{rk}\left(\operatorname{im} j_{i}\right) .
$$

Thus $\operatorname{rk}(\operatorname{im} \partial)=\operatorname{rk}\left(\operatorname{im} j_{i}\right)$. The middle three terms give that $\operatorname{rk}(\operatorname{im} \partial)+\operatorname{rk}\left(\operatorname{im} j_{i}\right)=$ $\operatorname{rk}\left(H_{q} M_{i}\right)$. Thus $\operatorname{rk}\left(K_{i}\right)=\operatorname{rk}(\operatorname{im} \partial)=\frac{1}{2} \operatorname{rk}\left(H_{q} M_{i}\right)$.

When $q \geq 2, C_{2 q-1}\left(B_{m}\right)$ is an abelian group under the boundary connected sum and we now have a homomorphism

$$
\phi(m, q): C_{2 q-1}\left(B_{m}\right) \rightarrow G\left(m,(-1)^{q}\right)
$$

defined by assigning to the boundary cobordism class of a Seifert surface, the cobordism class of the corresponding Seifert matrix.

Unfortunately, $C_{1}\left(B_{m}\right)$ does not have a group structure (see Lemma 2.10 and Proposition 2.11). But we still have $\phi(m, 1): C_{1}\left(B_{m}\right) \rightarrow G(m,-1)$ as a well-defined map of sets. In fact, let $M$ and $N$ be Seifert surfaces in $S^{3}$ whose Seifert matrices are $A$ and $B$ on a given basis of $H_{1} M$ and $H_{1} N$, respectively. Suppose that $M$ is boundary cobordant to $N$ by a boundary cobordism $I M$ in $S^{3} \times I$. After isotoping $I M$, we may choose a point $p \in S^{3}$ such that $p \times I$ in $S^{3} \times I$ does not intersect $I M$. Then the complement of an open tubular neighborhood of $p \times I$ in $S^{3} \times I$ is diffeomorphic to $D^{4}$. Consider the surfaces $\left(M_{1} \cup N_{1}\right) \cup \cdots \cup\left(M_{m} \cup N_{m}\right)$ in $D^{4}$. We may take the Seifert pairing on these surfaces which will give the matrix $A \oplus(-B)$ on the given basis of $H_{1}\left(M_{1} \cup N_{1}\right) \oplus \cdots \oplus H_{1}\left(M_{m} \cup N_{m}\right)$. Let $K_{i}$ be the kernel of $j_{i}: H_{1}\left(M_{i} \cup N_{i}\right) \rightarrow H_{1}\left(I M_{i}\right)$ where $j_{i}$ is induced from the inclusion. Then the Seifert pairing over $K_{1} \oplus \cdots \oplus K_{m}$ is zero by the same reason as in the proof of 3.3. At this time we take a boundary connected sum $M_{i} \downarrow N_{i}$ of $M_{i}$ and $N_{i}$ using a band in $\partial I M_{i}-\operatorname{int}\left(M_{i} \cup N_{i}\right)$. Then $\partial I M_{i}-\operatorname{int}\left(M_{i} \downarrow N_{i}\right)$ is homeomorphic to $D^{2}$. We can now use the argument in the proof of 3.3 to prove that $\operatorname{rk}\left(K_{i}\right)=\frac{1}{2} \operatorname{rk}\left(H_{1}\left(M_{i} \natural N_{i}\right)\right)$.

The following theorem asserts realizations of Seifert matrices (compare [Ke]). 
THEOREM 3.4. (1) For $q \neq 2$, let $A=\left(A_{i j}\right)$ be a Seifert matrix of type $\left(m,(-1)^{q}\right)$. Then there is a Seifert surface in $S^{2 q+1}$ with Seifert matrix $A$.

(2) Let $B=\left(B_{i j}\right)$ be a Seifert matrix of type $(m,+1)$ such that $B_{i i}+B_{i i}^{T}$ has signature a multiple of 16 for each $i=1, \ldots, m$. Then there is a Seifert surface in $S^{5}$ whose Seifert matrix is cobordant ${ }^{4}$ to $B$.

In fact, those Seifert surfaces are ones of simple links.

PROOF. (1) For $q=1$, this is essentially a classical construction-disk with bands (see [Se]). We assume $q \geq 3$. We build a $2 q$ dimensional handlebody $M_{i}$ such that

(i) $M_{i}$ has one 0 -handle and $n_{i} q$-handles so that the intersection pairing on $H_{q} M_{i}$ gives the matrix $A_{i i}+(-1)^{q} A_{i i}^{T}$ on the basis obtained from cores of $q$-handles;

(ii) $M_{i}$ is stably parallelizable.

We take a disk $D^{2 q}$ as a 0 -handle and choose framed embeddings $i_{r}: S^{q-1} \rightarrow D^{2 q}$ with framing $t_{r} \in \Pi_{q-1} \mathrm{SO}_{q}$ for $r=1, \ldots, n_{i}$ such that

(i) $\operatorname{lk}\left(i_{r}\left(S^{q-1}\right), i_{s}\left(S^{q-1}\right)\right)=a_{r s}^{i}+(-1)^{q} a_{s r}^{i}$ for $r \neq s$ where $a_{r s}^{i}$ is the $(r, s)$ th entry of $A_{i i}$;

(ii) $t_{r}=\partial\left(a_{r r}^{i}\right)$ where the $(r, r)$ th entry $a_{r r}^{i}$ of $A_{i i}$ is regarded as an element of $\Pi_{q} S^{q}$ and $\partial$ is the boundary map of the homotopy long exact sequence of the fibration $\mathrm{SO}_{q} \rightarrow \mathrm{SO}_{q+1} \rightarrow S^{q}$.

We add $n_{i} q$-handles on $D^{2 q}$ along these framed embeddings $\left(i_{r}, t_{r}\right)$ to get a handle body $M_{i}$. Since $t_{r}$ is in the kernel of the natural map: $\Pi_{q-1} \mathrm{SO}_{q} \rightarrow \Pi_{q-1} \mathrm{SO}$, $M_{i}$ is stably parallelizable $[\mathbf{K M}]$. We note that $p_{*}\left(t_{r}\right)=a_{r r}^{i}+(-1)^{q} a_{r r}^{i}$ where $p_{*}: \Pi_{q-1} \mathrm{SO}_{q} \rightarrow \Pi_{q-1} S^{q-1}$ is induced from the projection. Let $c_{r}^{i}$ be the homology class in $H_{q} M_{i}$ obtained by the union of the core of the $r$ th $q$-handle and a suitable embedded $q$-disk in $D^{2 q}$. Then it is clear for $r \neq s$ that $c_{r}^{i} \cdot c_{s}^{i}=a_{r s}^{i}+(-1)^{q} a_{s r}^{i}$. We note that $c_{r}^{i} \cdot c_{r}^{i}$ is the Euler number of the normal bundle of an embedded $q$-sphere representing $c_{r}^{i}$. So we have $c_{r}^{i} \cdot c_{r}^{i}=a_{r r}^{i}+(-1)^{q} a_{r r}^{i}$. Thus we have constructed the desired handlebody $M_{i}$.

Since the intersection matrix $A_{i i}+(-1)^{q} A_{i i}^{T}$ of $M_{i}$ is unimodular and $M_{i}$ is $(q-1)$-connected, $\partial M_{i}$ is a homology $(2 q-1)$-sphere. $\partial M_{i}$ is the result of index $q$ surgeries along $i_{r}: S^{q-1} \rightarrow D^{2 q}, r=1, \ldots, n_{i}$. But the complement of the union of images of $i_{r}, r=1, \ldots, n_{i}$ in $D^{2 q}$ is at least 1-connected by general position for $q \geq 3$. This claim obviously breaks down for $q=2$. Now Van Kampen's theorem tells us that $\partial M_{i}$ is 1-connected. Thus $\partial M_{i}$ is a homotopy $(2 q-1)$-sphere and hence is homeomorphic to $S^{2 q-1}$ for $q \geq 3$.

Let $Q_{i}$ be a $q$-skeleton of $M_{i}$ obtained by collapsing the 0 -handle to a point and $q$-handles to their cores. Let $M=M_{1} \cup \cdots \cup M_{m}$ and $Q=Q_{1} \cup \cdots \cup Q_{m}$. Since each $M_{i}$ is stably parallelizable, $M$ can be immersed into $S^{2 q+1}$. By isotoping this immersion slightly, we may assume that $Q$ is embedded in $S^{2 q+1}$. Thus a smooth regular neighborhood of $Q$ in $M$ can be taken to be embedded in $S^{2 q+1}$. Since the smooth regular neighborhood of $Q$ is diffeomorphic to $M$, we eventually have an embedding $f: M \rightarrow S^{2 q+1}$.

Let $A(f)=\left(A_{i j}(f)\right)$ be the Seifert matrix of the Seifert surface $f(M)$ with respect to the basis $\left\{f_{*}\left(c_{r}^{i}\right)\right\}_{i=1, \ldots, m ; r=1, \ldots, n_{i}}$. Then it should be held that

$$
A_{i i}+(-1)^{q} A_{i i}^{T}=A_{i i}(f)+(-1)^{q} A_{i i}(f)^{T} .
$$

\footnotetext{
${ }^{4}$ Cobordism can be replaced by a stronger relation, called $S$-equivalence [Ko].
} 
We denote the $(r, s)$ th entry of the $(i, j)$ th matrix of a Seifert matrix $A$ by $a_{r s}^{i j}$. Let $g: M \rightarrow S^{2 q+1}$ be an embedding with Seifert matrix $A(g)$. We will show how to change $g$ to get new embeddings:

(I) $g^{\prime}: M \rightarrow S^{2 q+1}$ with Seifert matrix $A\left(g^{\prime}\right)$ such that for given $i, j, r$ and $s$ with $i \geq j$ and $r>s, A\left(g^{\prime}\right)=A(g)$ except possibly the following three entries: $a_{r s}^{i j}\left(g^{\prime}\right)=a_{r s}^{i j}$ and $a_{r s}^{j i}\left(g^{\prime}\right)=a_{r s}^{j i}$ and $a_{r r}^{i i}\left(g^{\prime}\right)$ is undetermined;

(II) $g^{\prime \prime}: M \rightarrow S^{2 q+1}$ with Seifert matrix $A\left(g^{\prime \prime}\right)$ such that for given $i$ and $r$, $A\left(g^{\prime \prime}\right)=A(g)$ except $a_{r r}^{i i}\left(g^{\prime \prime}\right)=a_{r r}^{i i}$.

Starting with $f: M \rightarrow S^{2 q+1}$, we can have an embedding $f^{\prime}: M \rightarrow S^{2 q+1}$ such that its Seifert matrix matches with $A$ except diagonal entries through changes of type (I). Then using changes of type (II) on $f^{\prime}$, we eventually have an embedding of $M$ into $S^{2 q+1}$ whose Seifert matrix is exactly $A$.

In order to show (I), let $S$ be an embedded oriented $q$-sphere in $S^{2 q+1}-g(M)$ such that $S$ links only the $s$ th handle of $g\left(M_{j}\right)$ once, i.e., $\operatorname{lk}\left(S, g_{*}\left(c_{s}^{j}\right)\right)=1$. Let $d=$ $a_{r s}^{i j}-a_{r s}^{i j}(g)$. We embed $|d|$ parallel copies of $S$ in $S^{2 q+1}-g(M)$ and take a connected sum of these $S$ 's (or $-S$ 's if $d<0$ ) and denote it by $d S$. Let $h_{r}^{i}: D^{q} \times D^{q} \rightarrow M_{i}$ be an embedding whose image is exactly the $r$ th handle of $M_{i}$. We define an embedding $g^{\prime}: M \rightarrow S^{2 q+1}$ as follows:

$$
g^{\prime}=g \quad \text { on } M-h_{r}^{i}\left(\operatorname{int} D^{q} \times D^{q}\right)
$$

and

$$
g^{\prime}\left(h_{r}^{i}\left(D^{q} \times 0\right)\right)=g\left(h_{r}^{i}\left(D^{q} \times 0\right)\right) \# d S .
$$

Then we extend $g^{\prime}$ arbitrarily to be an embedding: $M \rightarrow S^{2 q+1}$. It is easy to check that the Seifert pairing on $g^{\prime}(M)$ gives the desired matrix on the basis $\left\{g^{\prime}\left(c_{r}^{i}\right)\right\}$.

Let $c_{r}^{i}$ itself denote an embedded $q$-sphere in $M_{i}$ representing the homology class $c_{r}^{i}$ of $H_{q} M_{i}$. Consider the following situation: Let $\varepsilon^{q+1}$ be the trivial $(q+1)$ dimensional bundle over $S^{q}$ and let $v$ be a vector field of this bundle. By choosing a trivialization of $\varepsilon^{q+1}, v$ determines an integer $[v]$ as an element of $\Pi_{q} G_{q+1,1}^{+} \cong \mathbf{Z}$. Then the $q$-dimensional bundle over $S^{q}$ given by the orthogonal complement of $v$ in $\varepsilon^{q+1}$ is identified with $\partial([v])$ where $\partial: \Pi_{q} S^{q} \rightarrow \Pi_{q-1} S_{q}$ is the boundary map. Furthermore, $\partial([v])$ does not depend on the choice of trivialization of $\varepsilon^{q+1}$ (see [Sd]). Now let $v$ be the vector field of the trivial normal 1-bundle of the embedding $g: M \rightarrow S^{2 q+1}$. Then $\partial\left(a_{r r}^{i i}(g)\right)$ can be identified with the orthogonal complement of $\left.v\right|_{c_{r}^{i}}$ in the trivial normal $(q+1)$-bundle of the embedding $g: c_{r}^{i} \rightarrow S^{2 q+1}$ which is isomorphic to the normal bundle of $c_{r}^{i}$ in $M_{i}$ by the bundle map $d g$. Since the normal bundle of $c_{r}^{i}$ in $M_{i}$ is identified with $\partial\left(a_{r r}^{i i}\right)$, we have $\partial\left(a_{r r}^{i i}-a_{r r}^{i i}(g)\right)=0$. This means that

$$
a_{r r}^{i i}-a_{r r}^{i i}(g)= \begin{cases}0, & q=\text { even, } \\ \text { even, } & q=\text { odd } \neq 3,7, \\ \text { arbitrary, }, & q=3,7 .\end{cases}
$$

Let $t:\left(D^{q}, S^{q-1}\right) \rightarrow\left(\mathrm{SO}_{q+1}\right.$, id $)$ be a smooth map with $p_{*}([t])=a_{r r}^{i i}-a_{r r}^{i i}(g)$ and $t \equiv$ id on a collar neighborhood of $S^{q-1}$. We define a new embedding $g^{\prime \prime}: M \rightarrow$ $S^{2 q+1}$ as follows: First

$$
g^{\prime \prime}=g \quad \text { on } M-h_{r}^{i}\left(\operatorname{int} D^{q} \times\left(D^{q}-0\right)\right) .
$$

Let $\nu$ be the trivial normal $(q+1)$-bundle of the restriction $g \mid h_{r}^{i}\left(D^{q} \times 0\right)$ in $S^{2 q+1}$. Then the restriction $v \mid h_{r}^{i}\left(D^{q} \times 0\right)$ is a vector field of this bundle. We define a new 
vector field $v^{\prime}$ of this bundle by

$$
v^{\prime}=t \cdot v \quad \text { on } h_{r}^{i}\left(D^{q} \times 0\right) .
$$

We extend $g^{\prime \prime}$ defined on $h_{r}^{i}\left(\partial D^{q} \times D^{q} \cup D^{q} \times 0\right)$ to a map $g^{\prime \prime}$ defined on $h_{r}^{i}\left(D^{q} \times D^{q}\right)$ by taking the orthogonal complement of the cross section given by $v^{\prime}$ in a tubular neighborhood $\nu$ of $g^{\prime \prime}\left(h_{r}^{i}\left(D^{q} \times 0\right)\right)$ as $g^{\prime \prime}\left(h_{r}^{i}\left(D^{q} \times D^{q}\right)\right)$. Then it is easy to see that the Seifert pairing on $g^{\prime \prime}(M)$ gives the desired matrix of (II) on the basis $\left\{g_{*}^{\prime \prime}\left(c_{r}^{i}\right)\right\}$.

PROOF OF (2). For $k=1, \ldots, m$ let $N_{k}=\left(N_{i j}\right)$ be a Seifert matrix of type $(m, \varepsilon)$ defined by

$$
N_{i j}= \begin{cases}\left(\begin{array}{ll}
0 & 1 \\
0 & 0
\end{array}\right), & \text { if } i=j=k, \\
\text { the matrix of size zero, }, & \text { otherwise. }\end{cases}
$$

We note that adding copies of $N_{1}, \ldots, N_{m}$ to a given Seifert matrix of type $(m, \varepsilon)$ does not change its cobordism class. Thus by adding enough copies of $N_{1}, \ldots, N_{m}$ any Seifert matrix $B=\left(B_{i j}\right)$ of type $(m,+1)$ is cobordant to a matrix $A=\left(A_{i j}\right)$ of the same type such that for all $i=1, \ldots, m, r_{i}$ is arbitrarily larger than $\sigma_{i}$ where $r_{i}$ and $\sigma_{i}$ are the rank and the signature of $A_{i i}+A_{i i}^{T}$.

According to $[\mathbf{M}]$, there is a closed 1-connected 4-manifold, called the Kummer surface $^{5}$, whose signature is exactly 16 and rank is 22 . Let $P$ be the punctured Kummer surface. According to $[\mathbf{A K}], P$ can be obtained by adding 2-handles to $D^{4}$ along a framed 22-link. Let $M_{i}$ be a boundary connected sum of $\sigma_{i} / 16$ copies of $P$ and $\frac{1}{2}\left(r_{i}-11 \sigma_{i} / 8\right)$ copies of punctured $S^{2} \times S^{2}$. Then $M_{i}$ is a 1-connected, stably parallelizable 4-manifold such that $\partial M_{i}$ is diffeomorphic to $S^{3}$ and $M_{i}$ has signature $\sigma_{i}$ and second Betti number $r_{i}$. Let $\left\{c_{r}^{i}\right\}$ be the basis of $H_{2} M_{i}$ given by the cores of 2 -handles of $M_{i}$. Since indefinite even unimodular symmetric matrices over $\mathbf{Z}$ are completely determined by their ranks and signatures $[\mathbf{M}]$, there is a unimodular matrix $U_{i}$ such that the intersection matrix of $M_{i}$ on the basis $\left\{c_{r}^{i}\right\}$ is given by $U_{i}\left(A_{i i}+A_{i i}^{T}\right) U_{i}^{T}$.

Let $M=M_{1} \cup \cdots \cup M_{m}$. As in the case $q \geq 3$, we can find an embedding $f: M \rightarrow S^{5}$ using the 2 -skeleton of $M$ given by cores of 2 -handles. Let $B=\left(B_{i j}\right)$ be the Seifert matrix of $f(M)$ in $S^{5}$ on the basis of $\left\{f_{*}\left(c_{r}^{i}\right)\right\}$. Then we should have $B_{i i}+B_{i i}^{T}=U_{i}\left(A_{i i}+A_{i i}^{T}\right) U_{i}^{T}$. Using the same argument as the case $q \geq 3$, we can repair the embedding $f$ on 2 -handles (no twisting on 2-handles is necessary because diagonal entries of Seifert matrices are determined by corresponding intersection matrices in this dimension) to get a new embedding $g: M \rightarrow S^{5}$ such that the Seifert matrix of $g(M)$ in the basis $\left\{g_{*}\left(c_{r}^{i}\right)\right\}$ is exactly $\left(U_{i} A_{i j} U_{j}^{T}\right)$.

Let $\Delta: G(m,+1) \rightarrow m(8 \mathbf{Z})$ be the map taking the signatures of $A_{i i}+A_{i i}^{T}$ for $i=1, \ldots, m$ for a Seifert matrix $\left(A_{i j}\right)$ of type $(m,+1)$. Then we define a subgroup $G^{0}(m,+1)$ of index $2^{m}$ of $G(m,+1)$ to be $\Delta^{-1}(m(16 \mathbf{Z}))$.

Our main theorem is now stated.

THEOREM 3.5. (1) $\phi(m, 1): C_{1}\left(B_{m}\right) \rightarrow G(m,-1)$ is a surjection.

(2) $\phi(m, 2): C_{3}\left(B_{m}\right) \rightarrow G^{0}(m,+1)$ is an isomorphism.

(3) $\phi(m, q): C_{2 q-1}\left(B_{m}\right) \rightarrow G\left(m,(-1)^{q}\right)$ is an isomorphism for $q \geq 3$.

PROOF. The ontonesses of the theorem follow from Theorem 3.4.

${ }^{5} \mathrm{This}$ is a nonsingular complex surface of degree 4 in $\mathrm{CP}^{3}$. 
We first show that the image of $\phi(m, 2)$ is contained in $G^{0}(m,+1)$. Let $M=$ $M_{1} \cup \cdots \cup M_{m}$ be a Seifert surface of dimension 4. $\partial M_{i}$ is homeomorphic to $S^{3}$ and so diffeomorphic to $S^{3}$. By putting a 4-disk on $\partial M_{i}$, we have a closed 4manifold $\hat{M}_{i}$. Let $A=\left(A_{i j}\right)$ be a Seifert matrix of $M$. Then $A_{i i}+A_{i i}^{T}$ represents the intersection pairing on $M_{i}$. Since each $\hat{M}_{i}$ is parallelizable, the signature of $A_{i i}+A_{i i}^{T}$ must be a multiple of 16 for each $i$ by Rochlin's theorem.

In order to prove that $\phi(m, q), q \geq 3$ is injective, it suffices by Lemma 2.8 to show that if $M=M_{1} \cup \cdots \cup M_{m}$ is a Seifert surface of dimension $2 q$ with the property that each $M_{i}$ is $(q-1)$-connected and $M$ has a null-cobordant Seifert matrix, then there is a submanifold $V=V_{1} \cup \cdots \cup V_{m}$ in $D^{2 q+2}$ as in Remark 2.12. Since $M$ has a null-cobordant Seifert matrix, we can choose for each $i=1, \ldots, m$ a basis $\left\{c_{i r}\right\}_{r=1, \ldots, 2 l_{i}}$ of $H_{q} M_{i}$ such that for $i, j=1, \ldots, m$ the Seifert pairing $\sigma\left(c_{i r}, c_{j s}\right)=0$ for $1 \leq r \leq l_{i}$ and $1 \leq s \leq l_{j}$. The intersection number $c_{i r} \cdot c_{i s}$ is identified with $\sigma\left(c_{i r}, c_{i s}\right)+(-1)^{q} \sigma\left(c_{i s}, c_{i r}\right)$, and hence they are zero for $1 \leq r, s \leq l_{i}$. Since $q \geq 3$, the Whitney's trick enables us to represent $c_{i 1}, \ldots, c_{i l_{i}}$ by disjoint embeddings of $S^{q}$ into $M_{i}$. We will essentially do framed surgeries on $M_{i}$ to kill $c_{i 1}, \ldots, c_{i l_{i}}$ and then the result of surgeries on $M_{i}$ is contractible as in [M1] and diffeomorphic to $D^{2 q}$ for $q \geq 3$.

But we want to have the traces $V_{i}, i=1, \ldots, m$, of the surgeries in the $D^{2 q+2}$, bounded by the ambient sphere $S^{2 q+1}$. So we need the following care. We embed $M \times I$ into $D^{2 q+2}$, for example, by pushing $M$ slightly into $D^{2 q+2}$ along the radial direction, so that

$$
(M \times I) \cap S^{2 q+1}=(M \times 0) \cap S^{2 q+1}=M .
$$

Let $\Delta_{i r}$ be an embedded $(q+1)$-disk in $D^{2 q+2}$ for each $i=1, \ldots, m$ and for each $r=1, \ldots, l_{i}$ such that $\partial \Delta_{i r}$ is an embedded $q$-sphere in $M_{i} \times 1$ representing $c_{i r}$ in $H_{q} M_{i}$ and int $\Delta_{i r}$ does not intersect $M \times I$. Since

$$
\Delta_{i r} \cdot \Delta_{j s}=\sigma\left(c_{i r}, c_{j s}\right)=0,
$$

we may assume by Whitney's trick that these disks are disjoint. Then these disks can be taken to be cores of $(q+1)$-handles to kill $c_{i r}$ 's. Each $\partial \Delta_{i r}$ has a normal 1 -frame in $D^{2 q+2}$ given by the positive normal direction of $M \times I$ in $D^{2 q+2}$. This 1 -frame can be considered as a cross section over $\partial \Delta_{i r}$ of the trivial normal bundle of $\Delta_{i r}$ in $D^{2 q+2}$ and then it determines a map from $\partial \Delta_{i r}$ to $G_{q+1,1}^{+} \cong S^{q}$ whose degree is identified with $\sigma\left(c_{i r}, c_{i r}\right)=0$. Thus the map is null homotopic and so can be extended to $\Delta_{i r}$, that is, we have a cross section over $\Delta_{i r}$ of the normal bundle of $\Delta_{i r}$ in $D^{2 q+2}$. Now we take the orthogonal complement of the cross section in the tubular neighborhood as a $q$-handle $H_{i r}$. Then it is easy to check that

$$
\left(M_{1} \times I \cup H_{11} \cup \cdots \cup H_{1 l_{1}}\right) \cup \cdots \cup\left(M_{m} \times I \cup H_{m 1} \cup \cdots \cup H_{m l_{m}}\right)
$$

has the properties of Remark 2.12 after smoothing the corners.

Now we prove that $\phi(m, 2)$ is injective. We first recall the following fact about 4-manifolds which can be easily seen through results in [W2].

Let $W$ be a closed 1-connected 4-manifold whose second Stiefel-Whitney class and signature are zero. Then for some $k \geq 0, W \# k\left(S^{2} \times S^{2}\right)$ is diffeomorphic to the boundary of a handle body with only one 0-handle and 2-handles.

Let $M=M_{1} \cup \cdots \cup M_{m}$ be a Seifert surface in $S^{5}$ whose Seifert matrix is null cobordant. Since we can assume that each $M_{i}$ is 1 -connected by Lemma 2.8, we 
may assume that each $M_{i}$ is diffeomorphic to the punctured boundary of such a handle body $H_{i}$ as above, after adding copies of $S^{2} \times S^{2}$ to $M_{i}$ inside $S^{5}$, which corresponds to adding Seifert matrices $N_{k}$ 's of the proof of 3.4 to the Seifert matrix of $M$. For $i=1, \ldots, m$ let $\left\{c_{i r}\right\}_{r=1, \ldots, 2 l_{i}}$ be a basis of $H_{2} M_{i}$ such that $\sigma\left(c_{i r}, c_{j s}\right)=0$ for all $i, j$ and $1 \leq r \leq l_{i}, 1 \leq s \leq l_{j}$. Since the signature of $M_{i}$ is zero, we may furthermore assume that for each $i=1, \ldots, m$ and $r, s=1, \ldots, l_{i}$,

$$
c_{i r} \cdot c_{i\left(l_{i}+s\right)}=\delta_{r s} \quad \text { and } \quad c_{i r} \cdot c_{i s}=0=c_{i\left(l_{i}+r\right)} \cdot c_{i\left(l_{i}+s\right)} .
$$

Let $k_{i}$ be the number of 2-handles in the handle body which was diffeomorphic to $M_{i}$. Let $S_{i\left(k_{i}+r\right)}^{\prime}$ for each $r=1, \ldots, k_{i}$ be an embedded 2 -sphere in $\partial H_{i}$ which is the boundary of the cocore of the $r$ th 2 -handle in $H_{i}$. And let $S_{i r}^{\prime}$ for $r=1, \ldots, k_{i}$ be an embedded 2-sphere in $\partial H_{i}$ which is parallel to the core of the $r$ th 2 -handle in $H_{i}$. We carry these embedded 2-spheres $S_{i r}^{\prime}$ in $H_{i}$ to embedded 2-spheres $S_{i r}$ in $M_{i}$ by the above diffeomorphism. Let $c_{i r}^{\prime}$ be the class in $H_{2} M_{i}$ given by $S_{i r}$ for $r=1, \ldots, 2 k_{i}$. Then it follows from a homology consideration that $k_{i}=l_{i}$ for all $i=1, \ldots, m$ and for $r, s=1, \ldots, l_{i}$

$$
c_{i r}^{\prime} \cdot c_{i\left(l_{i}+s\right)}^{\prime}=\delta_{r s} \quad \text { and } \quad c_{i r}^{\prime} \cdot c_{i s}^{\prime}=0=c_{i\left(l_{i}+r\right)}^{\prime} \cdot c_{i\left(l_{i}+s\right)}^{\prime} .
$$

Thus the automorphism of $H_{2} M_{i}$ sending $c_{i r}^{\prime}$ to $c_{i r}$ preserves the intersection pairing on $M_{i}$. According to [W1] there is a diffeomorphism $h_{i}$ of $M_{i}$ onto itself such that $\left(h_{i}\right)_{*}\left(c_{i r}^{\prime}\right)=c_{i r}$. Thus $c_{i r}$ can be represented by an embedded 2 -sphere $h_{i}\left(S_{i r}\right)$ in $M_{i}$. We can now perform surgeries on $M_{i}$ to kill $c_{i 1}, \ldots, c_{i l_{i}}$. Then the result of these surgeries on $M_{i}$ is diffeomorphic to $D^{4}$ because this is equivalent to adding 3 -handles on $H_{i}$ to cancel out all of the 2-handles and to taking the punctured boundary of the result which should be $D^{5}$.

We can now use the same technique as in the case $q \geq 3$ to have the traces of surgeries on $M_{1}, \ldots, M_{m}$ inside $D^{6}$.

We recall the $A_{m}$-action on $C_{n}\left(B_{m}\right)$ from $\S 2$ and carry this $A_{m}$-action over $G\left(m,(-1)^{q}\right)$ by the map $\phi(m, q)$ when $n=2 q-1$. Let $M=M_{1} \cup \cdots \cup M_{m}$ be a Seifert surface in $S^{2 q+1}$, and $A$ its Seifert matrix. Then $\phi(m, q)([M])=[A]$. Let $\alpha \in A_{m}$. Then the action by $\alpha$ on $[A]$ is in fact given by

$$
\alpha \cdot[A]=\phi(m, q)(\alpha \cdot[M])=\phi(m, q)(\{\alpha \cdot M]) .
$$

We will prove that this $A_{m}$-action on $G\left(m,(-1)^{q}\right)$ does not depend on the dimension $q$, but only on $(-1)^{q}$ by demonstrating this action explicitly.

Let $\alpha_{i j}$ be a generator of $A_{m}$ sending $x_{i}$ to $x_{j} x_{i} x_{j}^{-1}$ and fixing the other generators of $F_{m}$. We recall from $\S 2$ that

$$
\alpha_{i j} \cdot M=M_{1} \cup \cdots \cup M_{j-1} \cup M_{j}^{\prime \prime} \cup M_{j+1} \cup \cdots \cup M_{m}
$$

where $M_{j}^{\prime \prime}$ is a connected sum of $M_{j}$ and a double $M_{i}^{+} \cup M_{i}^{-}$of $M_{i}$. So $H_{q} M_{j}^{\prime \prime} \cong$ $H_{q} M_{j} \oplus H_{q} M_{i}^{+} \oplus H_{q} M_{i}^{-}$. For $s=1, \ldots, m$ let $\left\{c_{s \alpha}\right\}$ be a basis of $H_{q} M_{s}$ on which $M$ has the Seifert matrix $A=\left(A_{s t}\right)_{s, t=1, \ldots, m}$. Let $\left\{c_{i \alpha}^{+}\right\}$(or $\left\{c_{i \alpha}^{-}\right\}$, respectively) be the basis of $H_{q} M_{i}^{+}$(or $H_{q} M_{i}^{-}$) obtained by translating $\left\{c_{i \alpha}\right\}$ along the positive (or negative) direction of $M_{i}$. We compute the Seifert pairing on $\alpha_{i j} \cdot M$ with respect to the basis $\left\{c_{s \alpha}\right\}$ of $H_{q} M_{s}$ for $s \neq j$ and the basis $\left\{c_{j \alpha}\right\} \cup\left\{c_{i \alpha}^{+}\right\} \cup\left\{c_{i \alpha}^{-}\right\}$of $H_{q} M_{j}^{\prime \prime}$. For $s \neq i, \neq j$

$$
\sigma\left(c_{s \alpha}, c_{i \beta}^{+}\right)=\sigma\left(c_{s \alpha}, c_{i \beta}\right)=\sigma\left(c_{s \alpha}, c_{i \beta}^{-}\right)
$$


and

$$
\sigma\left(c_{i \alpha}, c_{i \beta}^{+}\right)=\sigma\left(c_{i \alpha}, c_{i \beta}\right), \quad \sigma\left(c_{i \alpha}, c_{i \beta}^{-}\right)=-(-1)^{q} \sigma\left(c_{i \beta}, c_{i \alpha}\right)
$$

and

$$
\sigma\left(c_{j \alpha}, c_{i \beta}^{+}\right)=\sigma\left(c_{j \alpha}, c_{i \beta}\right)=\sigma\left(c_{j \alpha}, c_{i \beta}^{-}\right) .
$$

For the following computation, we note that $M_{i}^{+}$(or $M_{i}^{-}$, respectively) has the negative (or positive) normal direction of $M_{i}$ as its positive normal direction. Then

$$
\sigma\left(c_{i \alpha}^{+}, c_{i \beta}^{+}\right)=-(-1)^{q} \sigma\left(c_{i \beta}, c_{i \alpha}\right)=\sigma\left(c_{i \alpha}^{+}, c_{i \beta}^{-}\right)
$$

and

$$
\sigma\left(c_{i \alpha}^{-}, c_{i \beta}^{+}\right)=\sigma\left(c_{i \alpha}, c_{i \beta}\right)=\sigma\left(c_{i \alpha}^{-}, c_{i \beta}^{-}\right) .
$$

Let $B^{\prime}=\left(B_{s t}^{\prime}\right)$ be the Seifert matrix of $\alpha_{i j} \cdot M$ with respect to the above basis. Then $B^{\prime}$ is given by

$$
\begin{aligned}
B_{j j}^{\prime} & =\left(\begin{array}{ccc}
A_{j j} & A_{j i} & A_{j i} \\
A_{i j} & -\varepsilon A_{i i}^{T} & -A_{i i}^{T} \\
A_{i j} & A_{i i} & A_{i i}
\end{array}\right), \\
B_{j i}^{\prime} & =\left(\begin{array}{c}
A_{j i} \\
-\varepsilon A_{i i}^{T} \\
A_{i i}
\end{array}\right), \quad B_{j t}^{\prime}=\left(\begin{array}{c}
A_{j t} \\
A_{i t} \\
A_{i t}
\end{array}\right) \quad \text { for } t \neq i, j, \\
B_{i j}^{\prime} & =\left(\begin{array}{lll}
A_{i j} & A_{i i} & -\varepsilon A_{i i}^{T}
\end{array}\right), \\
B_{s j}^{\prime} & =\left(\begin{array}{lll}
A_{s j} & A_{s i} & A_{s i}
\end{array}\right) \quad \text { for } s \neq i, j \\
B_{s t}^{\prime} & =A_{s t} \text { for } s \neq j \text { and } t \neq j
\end{aligned}
$$

where $\varepsilon=(-1)^{q}$. Then the Seifert matrix $B^{\prime}$ is congruent to a simpler Seifert matrix $B=\left(B_{s t}\right)$ given by

$$
\begin{aligned}
B_{j j} & =\left(\begin{array}{ccc}
A_{j j} & 0 & 0 \\
0 & 0 & -S_{i} \\
A_{i j} & 0 & 0
\end{array}\right), \\
B_{j i} & =\left(\begin{array}{c}
0 \\
-S_{i} \\
0
\end{array}\right), \quad B_{j t}=\left(\begin{array}{c}
A_{j t} \\
0 \\
A_{i t}
\end{array}\right) \quad \text { for } t \neq i, j, \\
B_{i j} & =\left(\begin{array}{lll}
0 & S_{i} & 0
\end{array}\right), \\
B_{s t} & =A_{s t} \text { for } s \neq j \text { and } t \neq j \\
B_{s j} & =\left(\begin{array}{lll}
A_{s j} & 0 & A_{s i}
\end{array}\right) \text { for } s \neq i, j
\end{aligned}
$$

where $S_{i}=A_{i i}+(-1)^{q} A_{i i}^{T}$ and 0's are zero matrices of suitable sizes. We will take the Seifert matrix $B$ above as a definition of the action by $\alpha_{i j}$ on a Seifert matrix $A$, i.e., $\alpha_{i j} \cdot A=B$.

It follows from Theorem 3.5 that this $A_{m}$-action on Seifert matrices is compatible with cobordisms of Seifert matrices. So we put

$$
\alpha_{i j} \cdot[A]=[B] \quad \text { in } G(m, \varepsilon) .
$$


Now we consider the set of boundary link cobordism classes of boundary $m$-links of dimension $n$, ignoring $F_{m}$-structures or choices of Seifert surfaces on those links. We denote the set by $B(n, m)$. By Lemma $2.3 B(n, m)$ is isomorphic to the orbit spaces $C_{n}\left(B_{m}\right) / A_{m}$ or $C_{n}\left(F_{m}\right) / A_{m}$ as sets. We note that $B(n, m)$ is not necessarily a group, even under our restricted connected sum operation. This corresponds to the fact that there is no reason for the orbit space $C_{n}\left(B_{m}\right) / A_{m}$ to be a group, although $C_{n}\left(B_{m}\right)$ is.

COROLlaRY 3.6. Let $G(m, \varepsilon) / A_{m}$ and $G^{0}(m,+1) / A_{m}$ be orbit spaces. Then we have the following periodicity of $B(n, m)$ :

For $n=2$ or $n \geq 4$,

$$
B(n, m) \cong \begin{cases}0, & n \equiv 0(\bmod 2) \\ G(m,-1) / A_{m}, & n \equiv 1(\bmod 4) \\ G(m,+1) / A_{m}, & n \equiv 3(\bmod 4)\end{cases}
$$

and $B(3, m) \cong G^{0}(m,+1) / A_{m}$.

Finally, we remark that $A_{2}$ is the trivial group because two generators $\alpha_{12}, \alpha_{21}$ of $C A_{2}$ are inner. Thus we have

$$
B(n, 2) \cong C_{n}\left(B_{2}\right) \cong C_{n}\left(F_{2}\right) .
$$

4. Examples. We give an example of a boundary link demonstrating that the action of the group $A_{m}$ on $C_{n}\left(B_{m}\right)$ or $C_{n}\left(F_{m}\right)$ is nontrivial. In our example, $m=3$. But one can easily generalize the example in various ways for all $m \geq 3$.

Let $B$ be the Seifert matrix of type $(2, \varepsilon)$ given by

$$
B=\left(\begin{array}{cc|cc}
0 & 1 & 1 & 0 \\
0 & -1 & 0 & 0 \\
\hline-\varepsilon & 0 & 0 & 1 \\
0 & 0 & 0 & -1
\end{array}\right)
$$

By Theorem 3.4, there is a Seifert surface $M^{\prime}$ in $S^{2 q+1}$ realizing $B, \varepsilon=(-1)^{q}$. When $q=2$, the Seifert matrix of $M^{\prime}$ is cobordant to $B$. Figure 1 illustrates $\partial M^{\prime}$ for $q=1$. We remark that the Seifert matrix $B$ is not null cobordant. So the boundary link $\partial M^{\prime}$ is not boundary slice. In fact, we can show, using the method in [Ko] and a theorem in [CS], that this link is not slice for $q \geq 3$.

We embed a $2 q$-disk $D$ missing $M^{\prime}$ in $S^{2 q+1}$. Consider the new Seifert surface $M=D \cup M^{\prime}$ in $S^{2 q+1}$. The Seifert matrix of $M$ is given by $A=(A i j)$ where $A i j$ are empty matrices for $i=1$ or $j=1$, and

$$
\left(\begin{array}{ll}
A_{22} & A_{23} \\
A_{32} & A_{33}
\end{array}\right)=B .
$$

Recall the action of the group $A_{m}$ on Seifert surfaces and on Seifert matrices. Let $\alpha_{21}$ be a generator of $A_{3}$ in Lemma 2.4. We will show that $\alpha_{21} \cdot M$ is not boundary cobordant to $M$ by proving that the Seifert matrix $\left(\alpha_{21} \cdot A\right) \oplus(-A)$ is not null cobordant. Thus the boundary connected sum $N=\left(\alpha_{21} \cdot M\right) \natural(-M)$ does not represent the identity element in $C_{2 q-1}\left(B_{3}\right)$. Figure 2 illustrates $\partial N$ for $q=1$. Since the action of $A_{m}$ on $C_{n}\left(B_{m}\right)$ fixes the identity element, $\alpha \cdot N$ does 


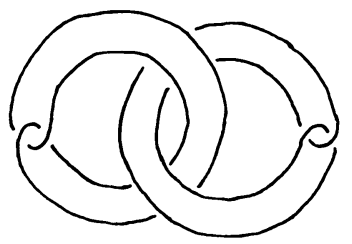

FIgURE 1

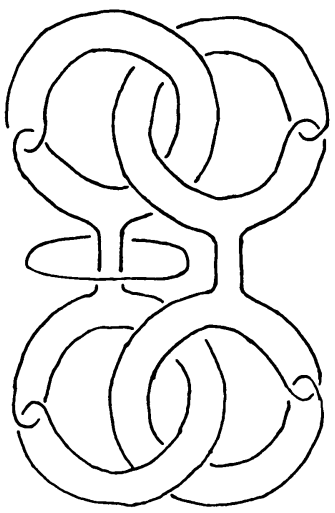

FIGURE 2

not represent the identity element for any $\alpha \in A_{3}$. Thus the boundary link $\partial N$ is not boundary slice for any choice of Seifert surfaces. But the question whether this link is slice, remains unanswered even though one of its obstructions [CS] to be slice vanishes due to its construction.

The Seifert matrix for $N$ is given by

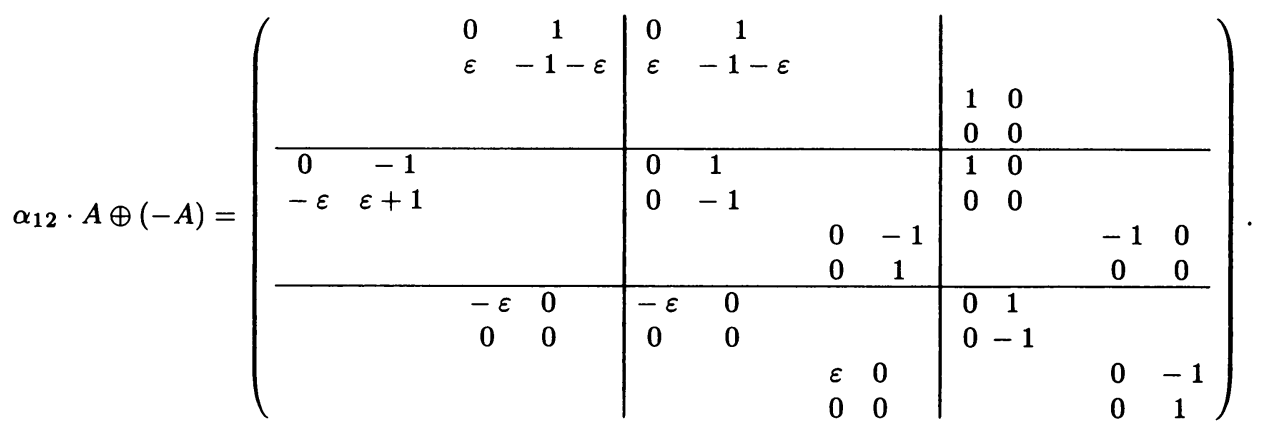

This matrix is cobordant (in fact, $S$-equivalent [Ko]) to

$$
C=\left(C_{i j}\right)=\left(\begin{array}{cc|cccc|ccccc}
0 & 1 & 1 & 0 & & & 0 & 0 & & \\
0 & 0 & 0 & 0 & & & 1 & 0 & & \\
\hline-\varepsilon & 0 & 0 & 1 & & & 1 & 0 & & \\
0 & 0 & 0 & -1 & & & 0 & 0 & & \\
& & & & 0 & -1 & & & -1 & 0 \\
& & & & 0 & 1 & & & 0 & 0 \\
\hline 0 & -\varepsilon & -\varepsilon & 0 & & & 0 & 1 & & \\
0 & 0 & 0 & 0 & & & 0 & -1 & & \\
& & & & \varepsilon & 0 & & & 0 & -1 \\
& & & & 0 & 0 & & & 0 & 1
\end{array}\right) .
$$

After tensoring with $\mathbf{Q}$, we consider $C_{i j}, i, j=1,2,3$ as a bilinear form

$$
C_{i j}: V_{i} \times V_{j} \rightarrow \mathbf{Q}
$$


where $V_{i}$ is a vector space over $\mathbf{Q}$ of rank 2 and $V_{2}, V_{3}$ are of rank 4. Let $\alpha_{i}$, $i=1, \ldots, 10$ be the basis for $V_{1} \oplus V_{2} \oplus V_{3}$ which gives the matrix representation $\left(C_{i j}\right)$ in this order.

Suppose that $\left(C_{i j}\right)$ is null cobordant. Then there are subspaces $K_{i}, i=1,2,3$ of $V_{i}$ satisfying

(i) $\operatorname{rk}\left(K_{i}\right)=\frac{1}{2} \operatorname{rk}\left(V_{i}\right)$

(ii) $C_{i j}\left(K_{i}, K_{j}\right)=0$, for all $i, j=1,2,3$.

By considering the matrix $C_{11}$, the basis element of $K_{1}$ should be either one of $\pm \alpha_{1}$ or $\pm \alpha_{2}$. Since $C_{22}$ and $C_{33}$ are identical, we may assume that $\alpha_{1}$ (or $-\alpha_{1}$ ) generates $K_{1}$. Let the linear combinations

$$
\beta_{i}=q_{i} \alpha_{3}+r_{i} \alpha_{4}+s_{i} \alpha_{5}+t_{i} \alpha_{6}, \quad i=1,2,
$$

be basis elements for $K_{2}$. Since $C_{12}\left( \pm \alpha_{1}, \beta_{i}\right)= \pm q_{i}, q_{i}=0$ for $i=1,2$.

Now $C_{22}\left(\beta_{i}, \beta_{j}\right)=0$ for $i, j=1,2$ give us four equations:

$$
\begin{aligned}
-r_{1}^{2}-s_{1} t_{1}+t_{1}^{2}=0 \cdots(\mathrm{i}), \\
-r_{2}^{2}-s_{2} t_{2}+t_{2}^{2}=0 \cdots \text { (ii), } \\
-r_{1} r_{2}-s_{1} t_{2}+t_{1} t_{2}=0 \cdots \text { (iii), } \\
-r_{2} r_{1}-s_{2} t_{1}+t_{2} t_{1}=0 \cdots \text { (iv). }
\end{aligned}
$$

We first show $t_{1} \neq 0$. If $t_{1}=0$, then $r_{1}=0$ by (i), then $s_{1} t_{2}=0$ by (iii). Since $\beta_{1}=0, s_{1} \neq 0$, so $t_{2}=0$, then $r_{2}=0$ by (ii). Thus we have $\beta_{1}=s_{1} \alpha_{5}$ and $\beta_{2}=s_{2} \alpha_{5}$. This is not possible because $\operatorname{rk}\left(K_{2}\right)=2$. Similarly we can show $t_{2} \neq 0$.

By (iii) and (iv), $s_{1} t_{2}=s_{2} t_{1}$ or $s_{1} / t_{1}=s_{2} / t_{2} \cdots(\mathrm{v})$. Also we have $s_{1} / t_{1}=$ $1-\left(r_{1} / t_{1}\right)^{2}$ by dividing (i) by $t_{1}^{2}$ and $s_{1} / t_{1}=1-\left(r_{1} / t_{1}\right)\left(r_{2} / t_{2}\right)$ by dividing (iii) by $t_{1} t_{2}$. Combining these two equations, $\left(r_{1} / t_{1}\right)\left(r_{1} / t_{1}-r_{2} / t_{2}\right)=0$. Thus we now have only two possibilities that $r_{1}=0$ or $r_{1} / t_{1}=r_{2} / t_{2}$.

If $r_{1}=0$, then $s_{1}=t_{1}$ by (i), then $s_{2}=t_{2}$ by (v), then $r_{2}=0$ by (ii). Thus we have $\beta_{1}=t_{1}\left(\alpha_{5}+\alpha_{6}\right)$ and $\beta_{2}=t_{2}\left(\alpha_{5}+\alpha_{6}\right)$. This is not possible because they are linearly dependent.

If $r_{1} / t_{1}=r_{2} / t_{2}$, then we have

$$
\beta_{1}=t_{1}\left(\frac{r_{1}}{t_{1}} \alpha_{4}+\frac{s_{1}}{t_{1}} \alpha_{5}+\alpha_{6}\right) \text { and } \beta_{2}=t_{2}\left(\frac{r_{1}}{t_{1}} \alpha_{4}+\frac{s_{1}}{t_{1}} \alpha_{5}+\alpha_{6}\right) .
$$

But this is not possible either.

Consequently, the Seifert matrix $C=\left(C_{i j}\right)$ cannot be null cobordant. Since the above argument does not depend on the matrix $C_{12}$ (or $C_{21}$ ), we may conclude that the following Seifert matrix of type $(3, \varepsilon)$ is not null cobordant:

$$
\left(\begin{array}{cc|cccc|cccc}
0 & 1 & M & 0 & M & 0 & 0 & 0 & 0 & 0 \\
0 & 0 & 0 & 0 & 0 & 0 & N & 0 & N & 0 \\
\hline-\varepsilon M & 0 & & & 1 & 0 & & & & \\
0 & 0 & & & 0 & 1 & & X & & \\
-\varepsilon M & 0 & & & & & & & & \\
0 & 0 & & & & & & & & \\
\hline 0 & -\varepsilon N & & & & & & 1 & 0 \\
0 & 0 & & & & & & & 0 & 1 \\
0 & -\varepsilon N & & -\varepsilon X^{T} & & & & & & \\
0 & 0 & & & & & & & &
\end{array}\right)
$$

where $M, N$ are arbitrary nonzero integers and $X$ is an arbitrary $(4 \times 4)$ matrix. 


\section{REFERENCES}

[AK] S. Akbulut and R. Kirby, Branched covers of surfaces in 4-manifolds, Math. Ann. 252 (1980), 111-131.

[Ca] S. Cappell, A splitting theorem for manifolds, Invent. Math. 33 (1976), 69-170.

[Co] T. Cochran, Slice links in $S^{4}$, Trans. Amer. Math. Soc. 285 (1984), 389-401.

[CS] S. Cappell and J. Shaneson, Link cobordism, Comment. Math. Helv. 55 (1980), 20-49.

[CS1] _ The codimension two placement problem and homology equivalent manifold, Ann. of Math. (2) 99 (1974), 277-348.

[De] R. DeMeo, Cobordisms of even dimensional links, Duke Math. J. 48 (1981), 23-33.

[Gu] M. Gutierrez, Boundary links and an unlinking theorem, Trans. Amer. Math. Soc. 171 (1972), 491-499.

[Gu1] _ Links of codimension two, Revista Columbiana de Matematicas, Monograf. Mat. 10 (1974). MR 54 \#3709.

[Hu] S. T. Hu, Homotopy theory, Academic Press, New York, 1959.

[K1] C. Kearton, Cobordism of knots and Blanchfield duality, J. London Math. Soc. 10 (1975), 406-408.

[K2] _ Classification of simple knots by Blanchfield duality, Bull. Amer. Math. Soc. 79 (1973), 406-408.

[Ke] M. Kervaire, Les noeuds de dimensions superieures, Bull. Soc. Math. France 93 (1965), 225-271.

[KM] M. Kervaire and J. Milnor, Groups of homotopy spheres. I, Ann. of Math. (2) 77 (1963), 504-537.

[Ko] K. H. Ko, Seifert matrices and boundary links, Thesis, Brandeis University, 1984.

[L1] J. Levine, Unknotting spheres in codimension two, Topology 4 (1965), 9-16.

[L2] _ Polynomial invariant of knots of codimension two, Ann. of Math. (2) 84 (1966), $537-554$.

[L3] _ Knot cobordism groups in codimension two, Comment. Math. Helv. 44 (1969), 229244.

[L4] _ Invariants of knot cobordism, Invent. Math. 8 (1969), 98-110.

[L5] _ An algebraic classification of some knots of codimension two, Comment. Math. Helv. 45 (1970), 185-198.

[L6] _ Knot modules. I, Trans. Amer. Math. Soc. 229 (1977), 1-50.

[M] J. Milnor, On simply-connected 4-manifolds, Sympos. Topologia Algebrica, Mexico, 1958, pp. $122-128$.

[M1] _ A procedure for killing the homotopy groups of differentiable manifolds, Proc. Sympos. Pure Math., vol. 3, Amer. Math. Soc., Providence, R.I., 1969, pp. 39-55.

[Mc] S. Mac Lane, Homology, Springer-Verlag, Berlin and New York, 1975.

[Or] K. Orr, New link invariants and applications, Thesis, Rutgers University, 1985.

[Ra] A. Ranicki, Exact sequence in the algebraic theory of surgery, Princeton Univ. Press, Princeton, N.J., 1981.

[Sa] N. Sato, Cobordisms of semi-boundary links, Topology and its Applications 18 (1984), $225-234$.

[Sd] N. Steenrod, The topology of fibre bundles, Princeton Univ. Press, Princeton, N.J., 1951.

[Se] H. Seifert, Über das Geschlecht von Knoten, Math. Ann. 110 (1934), 571-592.

[Sh] J. Shaneson, Non-simply-connected surgery and some results in low dimensional topology, Comm. Math. Helv. 45 (1970), 333-352.

[Sm] N. Smythe, Boundary links, Wisconsin Topology Seminar (1965), Ann. of Math. Studies, no. 60, Princeton Univ. Press, Princeton, N.J.

[St] J. Stallings, Homology and lower central series of groups, J. Algebra 2 (1965), 170-181.

[W1] C. T. C. Wall, Diffeomorphisms of 4-manifolds, J. London Math. Soc. 39 (1964), 131-140.

[W2] _ - On simply-connected 4-manifolds, J. London Math. Soc. 39 (1964), 141-149. 78712

Department of mathematics, University of Texas at Austin, Austin, Texas

Department of mathematics, Korea institute of Technology, Taejon, 30001, KOREA 